\title{
Tuberculosis vaccine development: from classic to clinical candidates
}

\author{
Junli Li ${ }^{1,2,3,4}$ (D) Aihua Zhao ${ }^{5}$ - Jun Tang ${ }^{1,2,3,4} \cdot$ Guozhi Wang ${ }^{5} \cdot$ Yanan Shi ${ }^{1,2,3,4} \cdot$ Lingjun Zhan $^{1,2,3,4} \cdot$ Chuan Qin $^{1,2,3,4}$
}

Received: 2 December 2019 / Accepted: 5 February 2020 / Published online: 15 February 2020

(C) Springer-Verlag GmbH Germany, part of Springer Nature 2020

\begin{abstract}
Bacillus Calmette-Guérin (BCG) has been in use for nearly 100 years and is the only licensed TB vaccine. While BCG provides protection against disseminated TB in infants, its protection against adult pulmonary tuberculosis (PTB) is variable. To achieve the ambitious goal of eradicating TB worldwide by 2050 , there is an urgent need to develop novel TB vaccines. Currently, there are more than a dozen novel TB vaccines including prophylactic and therapeutic at different stages of clinical research. This literature review provides an overview of the clinical status of candidate TB vaccines and discusses the challenges and future development trends of novel TB vaccine research in combination with the efficacy of evaluation of TB vaccines, provides insight for the development of safer and more efficient vaccines, and may inspire new ideas for the prevention of TB.
\end{abstract}

Keywords $\mathrm{MTB} \cdot \mathrm{TB} \cdot \mathrm{BCG} \cdot$ Novel TB vaccine $\cdot$ Clinical trial

\section{Introduction}

Tuberculosis (TB), caused by the human pathogens Mycobacterium tuberculosis (MTB), has plagued humanity for millennia and remains the deadliest infectious disease in the modern world [1]. Causes of TB resurgence are complex and include the emergence and prevalence of drug-resistant strains, low early detection rates, latent infection, poor public health care conditions, and population movements $[2,3]$. In 2018, there were more than 1.2 million TB deaths worldwide

Lingjun Zhan

zhanlingjun2008@163.com

Chuan Qin

qinchuan@pumc.edu.cn

1 NHC Key Laboratory of Human Disease Comparative Medicine, Institute of Laboratory Animal Sciences, Chinese Academy of Medical Sciences (CAMS) and Peking Union Medical College (PUMC), Beijing 100021, People's Republic of China

2 Beijing Key Laboratory for Animal Models of Emerging and Reemerging Infectious, Beijing 100021, People's Republic of China

3 Key Laboratory of Human Diseases Animal Model, State Administration of Traditional Chinese Medicine, Beijing 100021, People's Republic of China

4 Tuberculosis Center, Chinese Academy of Medical Sciences (CAMS), Beijing 100021, People's Republic of China

5 Division of Tuberculosis Vaccines, National Institutes for Food and Drug Control (NIFDC), Beijing 102629, People's Republic of China and an additional 251,000 deaths of TB-HIV co-infection. New TB cases reached 10 million (equivalent to 133 new cases per 100,000), and about $3.4 \%$ of new TB cases and $18 \%$ of previously treated cases had multi-drug resistant TB (MDR-TB) or rifampicin-resistant TB (RR-TB). Adult male patients accounted for $57 \%$ of all new-onset patients, and children younger than 15 years old and those with HIV coinfection accounted for $11 \%$ and $8.6 \%$ of new-onset patients, respectively [4].

In 1993, the World Health Organization (WHO) announced that TB is a global health emergency [5]. With social and economic development, TB morbidity and mortality in Western Europe, North America, and other developed regions began to decline in the early twentieth century. Currently, the number of new cases and deaths per 100,000 population are less than ten cases and one case, respectively [6,7]. However, for many countries, the major public health issue of ending TB remains a distant reality. At present, there are an estimated 1.7 billion latent tuberculosis infections (LTBI) worldwide, accounting for about a quarter of the population $[8,9]$. Given the increase of population movements and immigration, the need for prevention and control of TB worldwide is becoming more serious. Therefore, the 2017 G20 Leaders' Declaration listed the "End TB Strategy" as a key priority for global political attention.

Crucial strategies for controlling and eradicating TB include diagnosis, prevention, and treatment. Timely diagnosis and effective treatment play important roles for controlling the epidemic. In order to reduce incidences of TB in 2030 and 2035 
by 80 and $90 \%$ compared with levels in 2015 , its global incidence will need to decline at an unprecedented rate after 2025 . We therefore need new interventions, including shorter, less toxic treatments, improved diagnostics, and more effective vaccines. The TB vaccination is not only the most cost-effective method of controlling the disease but also allows TB outbreaks to be controlled at the source and must be a key component of any strategy to eliminate TB's global burden [10].

\section{Classic TB vaccine-BCG}

BCG is a live, attenuated vaccine that is obtained by continuous subculture of Mycobacterium bovis and is currently the only licensed vaccine for human TB prevention [11]. The BCG vaccine strain was originally distributed by the Institut Pasteur in France. Due to different passages and culture methods over the years, more than 14 BCG strains using different phenotypes and genotypes have been developed around the world [12-14] (Fig. 1). Currently, more than 90\% of the BCG used worldwide has been prepared from four major strains, which are BCG Pasteur 1173P2, BCG Danish 1331, BCG Glaxo 1077, and BCG Japan 172 strain. The BCG strain used for production in China is a progeny strain obtained by subculture of the BCG Danish 823 strain, introduced from the Statens Serum Institut in 1948. In 1992, the National Health and Commission of the People's Republic of China announced that the Bacillus $\mathrm{D}_{2} \mathrm{~PB} 302$ strain was the only $\mathrm{BCG}$ strain being produced in China (Fig. 1).

Since the early 1970s, BCG has been used as part of WHO's Expanded Programme on Immunization (EPI), which has used more than four billion doses to date and is the most widely used vaccine in history. In 1978, China began to carry out child immunization work nationwide, using a timely and effective BCG vaccination for newborn babies in accordance with prescribed immunization procedures. WHO recommends that newborns receive a single-dose BCG vaccination in high-burden countries, while low-burden countries can selectively vaccinate neonates. It is also recommended that BCG should be given to people who live in high-endemic areas or exposed to MDR-TB but are tuberculin-negative and BCG naïve.

BCG has been shown to be effective for preventing disseminated TB such as miliary TB and tuberculous meningitis in children [15]. In addition, BCG is considered to have a nonspecific effect, and it is associated with a decrease in infant mortality $[16,17]$. However, others have found that BCG is not significant for reducing TB overall incidence worldwide [18]. The effects of BCG vary widely, with $0-80 \%$ effectiveness in different settings, and vaccine-induced immunity diminishes over time. BCG provides 10-20 years of protection $[19,20]$, but does not provide adequate prevention of PTB for adolescents and adults [21-25].

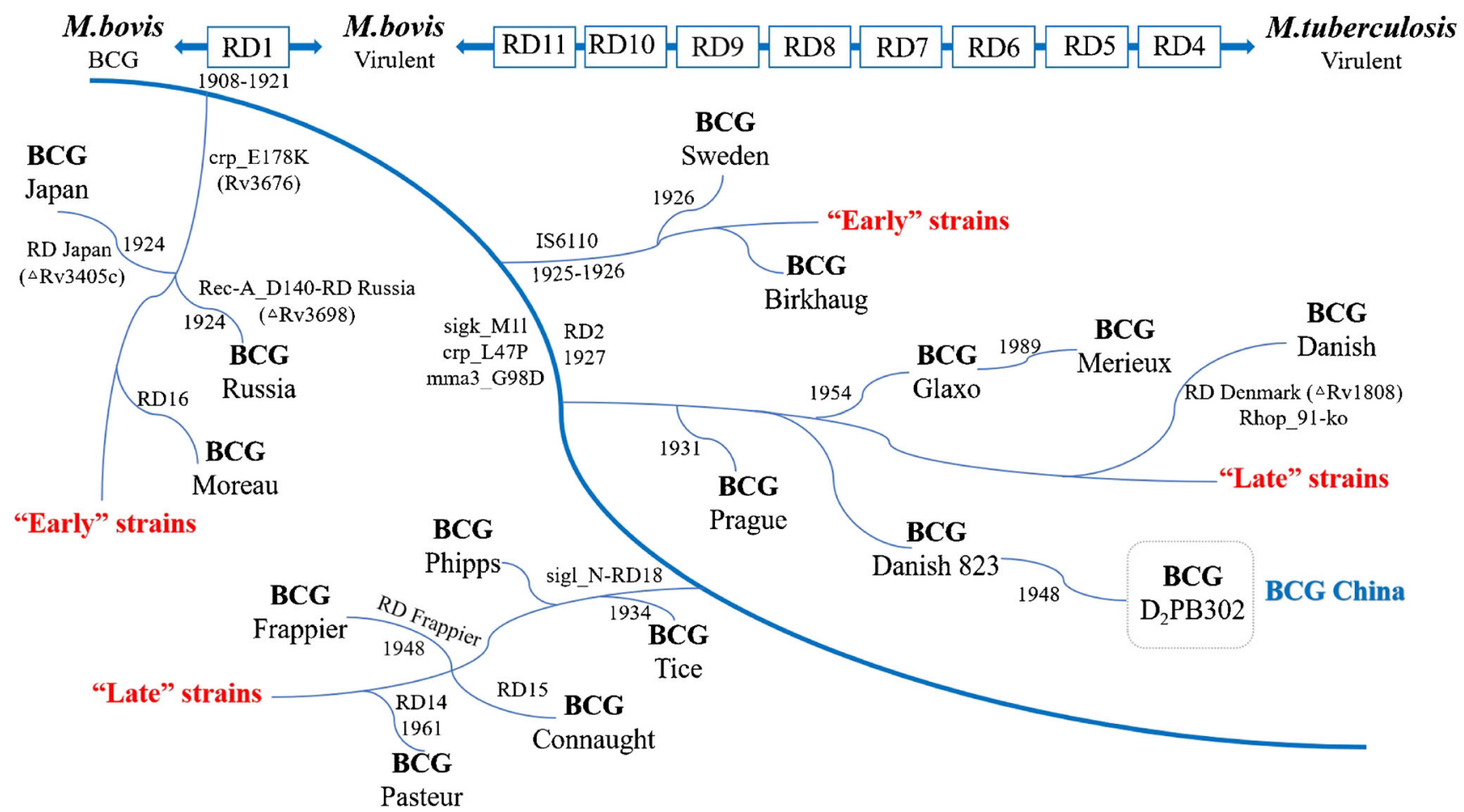

Fig. 1 History and genealogy of BCG substrains. RD1 was missing from all BCG substrains and coincides with the attenuated virulence of $M$. bovis. From the top to the bottom of the timeline, BCG substrains are divided into "Early" strains and "Late" strains. In "Early" strains, the

original characteristics of "authentic Pasteur" were conserved with fewer deletions, insertions, and mutations in the genome of the bacilli than the "Late" strains 
In recent years, it has been found that effectiveness evaluation of BCG is influenced by the screening of the tuberculin skin test (TST) prior to immunization. Higher vaccine efficacy was obtained for neonates and adult subjects who were screened by rigorous TST trials. BCG has a $59 \%$ protective effect against PTB in newborns (RR 0.41, 95\% CI: 0.29-0.58) and $74 \%$ in adult subjects who have undergone strict TST screening (RR 0.26, 95\% CI: 0.18-0.37). However, the average protection of PTB was significantly reduced in those who did not undergo strict TST screening [21].

BCG is suitable for people who are not sensitized by mycobacteria, although the incidence of LTBI is $60-70 \%$ in adults older than 25 in high-endemic areas [26], explaining why the BCG protection effect is low in areas with a high incidence of TB. The early South Indian Chingleput BCG clinical trial failed as most subjects were sensitized by MTB and non-tuberculous mycobacteria (NTM) [27, 28]. In short, BCG is suitable for uninfected people, cannot provide lifelong immunity, and is not suitable for HIV-infected patients and its preventive effect on TB needs to be correctly evaluated. However, so far, no new TB vaccine more protective than BCG has passed clinical assessment [29, 30]. Therefore, $\mathrm{BCG}$ remains one of the most cost-effective means of preventing TB in countries with high burden of TB for a long time.

\section{Candidate TB vaccines}

Despite high rates of $B C G$ vaccination as part of the EPI, the slow decline in global TB highlights the urgency of novel TB vaccines. Under the "End TB Strategy," developing new vaccines that reduce the prevalence of infection as well as the risk of TB will be a key element of achieving the goal of eradicating TB by 2050. By August 2019, more than 14 TB vaccines had been used in clinical trials, representing a diverse repertoire of formulations and mycobacterial antigens and that induce a wide range of immune responses with different characteristics (Table 1).

\section{Viral vector vaccines}

\section{Ad5 Ag85A}

This is a type 5 serotype replication-deficient adenoviral vector vaccine, which expresses the MTB Ag85A antigen and is designed to be a heterologous booster vaccine following $\mathrm{BCG}$ priming [31-34]. The target population is healthy adults following BCG vaccination. In a mouse model, a single intranasal immunization using $\mathrm{Ad} 5 \mathrm{Ag} 85 \mathrm{~A}$ induced an effective protection against the challenge of MTB, while, compared with an intramuscular injection, $\mathrm{Ad} 5 \mathrm{Ag} 85 \mathrm{~A}$ intranasal immunization can significantly enhance BCG's protective effect [35].
Phase I clinical trial (ClinicalTrials.gov Identifier: NCT00800670) has evaluated the safety and immunogenicity of Ad5 Ag85A in healthy volunteers who had a history of BCG vaccination and those BCG naïve. Overall, intramuscular vaccination was well tolerated and had good safety and immunogenicity for both groups, while a stronger multifunctional $\mathrm{T}$ cell response was observed in those with a history of BCG vaccination [36-38]. In September 2017, another phase I clinical trial (ClinicalTrials. gov Identifier: NCT02337270) is underway to evaluate the safety and immune responses of peripheral blood and lungs after one or two doses of Ad5Ag85A administered by aerosol in healthy volunteers previously immunized with $\mathrm{BCG}$, and the study is expected to be completed on April 2021.

\section{ChAd0X1 85A-MVA85A}

ChAdOx1 $85 \mathrm{~A}$ is a chimpanzee adenovirus [39], while MVA85A is a modified vaccinia Ankara virus, both of which express the MTB antigen Ag85A [40]. In preclinical study, ChAdOx1 85A immunogenicity and protective efficacy against MTB challenge were assessed in a mouse model. Researcher found that intranasally administered ChAdOx1 $85 \mathrm{~A}$ induced stronger $\mathrm{CD}^{+}$than $\mathrm{CD}^{+}{ }^{+} \mathrm{T}$ cell immune responses in both lungs and spleens, although it failed to protect mice against aerosol MTB infection. However, further boosting with MVA85A improves immunogenicity and protective efficacy in BCG vaccination [39]. Therefore, novel viral vector vaccines ChAdOx1 85A and MVA85A were used together to form a combined heterologous primary booster regimen and were administered via the mucosal route.

Currently, phase I trial (ClinicalTrials.gov Identifier: NCT01829490 and NCT03681860) of intramuscular injection of ChAdOx1 85A either alone or in combination with MVA85A for prime-boost regimen have been completed in the UK. Another phase I clinical trial (ClinicalTrials.gov Identifier: NCT04121494) designed to assess the safety and immunogenicity profile of ChAdOx1 85A given by aerosol inhaled versus intramuscular in adult healthy volunteers was also launched in January 2019 in Switzerland.

\section{TB/FLU-04L}

TB/FLU-04L is a replication-deficient attenuated influenza virus mucosal vector vaccine, which expresses the MTB antigens Ag85A and ESAT-6 and was developed by the Research Institute for Biological Safety Problems, Kazakhstan, in collaboration with the Research Institute on Influenza, Russia. It was designed as a preventive booster vaccine for infants, adolescents, and adults. In a preclinical mouse model, an intranasal boost of TB/FLU-04L can significantly improve the protective efficacy of BCG [41]. In phase I clinical trial (ClinicalTrials.gov Identifier: NCT02501421), 36 healthy 
Table 1 Summary of TB vaccine candidates currently under clinical assessment

\begin{tabular}{|c|c|c|c|c|}
\hline Strategy & Vaccine candidate & Vaccine type & Phase & Sponsor \\
\hline \multirow[t]{2}{*}{ Prime } & MTBVAC & $\begin{array}{l}\text { Live genetically attenuated } \\
\text { Mycobacterium tuberculosis }\end{array}$ & IIa & $\begin{array}{l}\text { University of Zaragoza; Biofabri; Tuberculosis } \\
\text { Vaccine Initiative }\end{array}$ \\
\hline & VPM1002 & $\begin{array}{l}\text { Live recombinant } \\
\text { Mycobacterium bovis }\end{array}$ & III & $\begin{array}{l}\text { Serum Institute of India; Vakzine Projekt } \\
\text { Management; Tuberculosis Vaccine Initiative; } \\
\text { Max Planck Institute for Infection Biology }\end{array}$ \\
\hline \multirow[t]{8}{*}{ Prime-boost } & Ad5 Ag85A & Viral vector & I & $\begin{array}{l}\text { McMaster University; Canadian Institutes of } \\
\text { Health Research; CanSino }\end{array}$ \\
\hline & ChAdOx1 85A-MVA85A & Viral vector & I & Oxford University \\
\hline & ID93 + GLA-SE & Protein/adjuvant & IIa & $\begin{array}{l}\text { Infectious Disease Research Institute; Aeras; } \\
\text { Wellcome Trust; International AIDS Vaccine } \\
\text { Initiative }\end{array}$ \\
\hline & TB/FLU-04L & Viral vector & IIa & $\begin{array}{l}\text { Research Institute for Biological Safety Problems; } \\
\text { Ministry of Health, Kazakhstan; Research } \\
\text { Institute of Influenza, Russia }\end{array}$ \\
\hline & $\begin{array}{l}\text { BCG revaccination } \\
\quad(\text { Gates MRI-TBV01-201) }\end{array}$ & Live attenuated Mycobacterium bovis & $\mathrm{IIb}$ & Bill \& Melinda Gates Medical Research Institute \\
\hline & DAR-901 booster & $\begin{array}{l}\text { Mycobacterium obuense- } \\
\text { whole cell or extract }\end{array}$ & $\mathrm{IIb}$ & $\begin{array}{l}\text { Dartmouth University; Global Health Innovative } \\
\text { Technology Fund; Aeras }\end{array}$ \\
\hline & H56:IC31 & Protein/adjuvant & $\mathrm{IIb}$ & Statens Serum Institut; Valneva; Aeras \\
\hline & M72/AS01 $($ GSK 692342) & Protein/adjuvant & $\mathrm{IIb}$ & Glaxo-SmithKline; Aeras \\
\hline \multirow[t]{4}{*}{ Immunotherapeutic } & $\mathrm{AEC} / \mathrm{BC} 02$ & Protein/adjuvant & I & AnHui Zhifei Longcom \\
\hline & RUTI $^{\circledR}$ & $\begin{array}{l}\text { Mycobacterium tuberculosis- } \\
\text { whole cell or extract }\end{array}$ & III & Archivel Farma S.L. \\
\hline & MIP/Immuvac & $\begin{array}{l}\text { Mycobacterium indicus pranii- } \\
\text { whole cell or extract }\end{array}$ & III & $\begin{array}{l}\text { Indian Council of Medical Research; Cadila } \\
\text { Pharmaceuticals }\end{array}$ \\
\hline & Vaccae $^{\mathrm{TM}}$ & $\begin{array}{l}\text { Mycobacterium vaccae- } \\
\text { whole cell or extract }\end{array}$ & III & AnHui Zhifei Longcom \\
\hline
\end{tabular}

Viral vectors: $A d 5$ adenovirus 5, ChAd chimpanzee adenovirus, $M V A$ modified vaccinia Ankara virus, $F L U$ replication-deficient influenza virus (H1N1) Adjuvants: GLA-SE oil in water emulsion/TLR4 agonist, IC31 cationic peptide/TLR9 agonist, $A S 01_{E}$ liposome/TLR4 agonist, BC02 BCG-CpG-DNA and $\mathrm{Al}(\mathrm{OH})_{3}$ compound adjuvant/TLR9 agonist

adults aged between 18 and 50 were vaccinated with TB/FLU04L on days 1 and 21, respectively, and results showed good safety and immunogenicity. Currently, the vaccine is undergoing phase IIa clinical trial for LTBI.

\section{Protein/adjuvant subunit vaccines}

\section{AEC/BC02}

$\mathrm{AEC} / \mathrm{BC} 02$ is a freeze-dried recombinant TB vaccine developed by the National Institutes for Food and Drug Control, China, and manufactured by Anhui Zhifei Longcom Biologic Pharmacy Co., Ltd. It is composed of MTB subunit protein, Ag85B; fusion protein, ESAT6-CFP10; and a compound adjuvant, BC02, based on BCG-derived unmethylated cytosinephosphate-guanine ( $\mathrm{CpG}$ ) DNA fragment and aluminum salt. It is mainly used for preventive treatment of LTBI. Preclinical studies have found that $\mathrm{AEC} / \mathrm{BC} 02$ can induce long-term antigen-specific cellular immune responses in mice, produce a therapeutic effect, and reduce the risk of Koch phenomenon in guinea pig LTBI model [42]. In April 2018, AEC/BC02 began a phase I clinical trial (ClinicalTrials.gov Identifier: NCT03026972) of human tolerance in four different populations. (1). Population I has 25 subjects who received TB-PPD skin test and IFN- $\gamma$ detection whose results are both negative. (2). Population II has 30 subjects who received TBPPD and ESAT6-CFP10 skin test in different arms and IFN- $\gamma$ detection whose results are all negative. (3). Population III as uninfected TB-PPD positive population, this group screened 30 subjects whose ESAT6-CFP10 skin test and IFN- $\gamma$ detection results are both negative, but TB-PPD skin test positive. (4). Population IV has 50 subjects whose three kinds of detection results are all positive (TB-PPD, ESAT6-CFP10, and IFN- $\gamma$ ). Currently, clinical volunteers are being recruited.

\section{H56: IC31}

H56: IC31 (AERAS-456) is a protein adjuvant vaccine consisting of MTB early secretory proteins Ag85B and ESAT-6 and latent infection-associated protein Rv2660c in combination with IC31 adjuvant. It is designed to induce immunity against new infections and reactivation of LTBI [43]. 
When compared with BCG, H56/IC31 vaccine can significantly reduce lung pathological damage and MTB extrapulmonary transmission while inducing a strong memory immune response. More importantly, H56: IC31-vaccinated non-human primates did not reactivate host latent-infected MTB following anti-TNF antibody treatment [44]. The vaccine has been used in three phase I clinical trials. Two of these were carried out with adults who were HIV-negative, with or without latent TB infection (ClinicalTrials.gov Identifier: NCT01967134 and NCT02378207) [43, 45, 46]. Another was conducted with HIV-negative, post-treatment patients with drug-susceptible PTB (ClinicalTrials.gov Identifier: NCT02375698), and it was found that the vaccine is safe and has good immunogenicity at all study doses. In addition, phase II trial (ClinicalTrials.gov Identifier: NCT03512249) assessing H56:IC31 for reducing the rate of TB disease recurrence in HIV-negative adults successfully treated for drug-susceptible PTB is ongoing in South Africa and the United Republic of Tanzania.

\section{ID93 + GLA-SE}

This is a protein adjuvant vaccine developed by the American Infectious Disease Research Institute in collaboration with Aeras. It consists of four MTB antigens that are associated with virulence (Rv2608, Rv3619, and Rv3620) or latent (Rv1813) as well as TLR-4 ligand GLA-SE adjuvant [47, 48]. In preclinical studies, the ID93 + GLA-SE vaccine has been found to be effective for preventing infection of sensitive and resistant MTB in mice and guinea pigs [49-52]. As a therapeutic vaccine, it can significantly improve the effect of anti-TB antibiotics in mice and non-human primates [53]. Additionally, the study also found that guinea pigs immunized with ID93 + GLA-SE showed a negative DTH response to intradermal PPD, which, in turn, did not interfere with the auxiliary diagnosis of a PPD skin test [54].

The vaccine has been used in two phase I clinical trials in the USA and South Africa. A phase I clinical trial (ClinicalTrials. gov Identifier: NCT01599897) in the USA included 60 healthy adults with no history of BCG vaccination who were negative for QuantiFERON-TB Gold and were intramuscularly injected with 3-needle ID93 and ID93 + GLA-SE vaccine. Researcher found that all dose levels of ID93 and ID93 + GLA-SE were acceptably safe. Compared with ID93 alone, this vaccine elicited higher titers of ID93-specific antibodies, preferentially increasing IgG1 and IgG3 antibody subclass and significantly inducing a multifunctional cytokine profile of $\mathrm{CD}^{+} \mathrm{T}$ cells [55]. Another phase Ib trial (ClinicalTrials.gov Identifier: NCT01927159) from South Africa also found that ID93 + GLA-SE was well tolerated, with no serious vaccine-related adverse events and vaccination-induced long-lasting antigenspecific IgG and Th1 cell responses, and that effects peaked after a second vaccination. At the same time, vaccine doses did not affect the frequency or severity of adverse events, while mild injection-site adverse reactions and flu-like symptoms were common in MTB-infected subjects compared with those not infected with MTB [56]. In addition, the USA (ClinicalTrials. gov identifier: NCT03722472) and South Korea (ClinicalTrials. gov identifier: NCT03806699) are also undergoing two phase I clinical trials in healthy adults and BCG-vaccinated adolescent, both of which are expected to be completed in 2020. In January 2017, a phase IIa clinical trial (ClinicalTrials.gov Identifier: NCT02465216) of the ID93 + GLA-SE vaccine was carried out in South Africa to assess the safety and immunogenicity of TB-HIV co-infected adults following treatment completion. Phase IIb clinical trial in the same population intended to prevent TB recurrence are being prepared.

\section{M72/AS01 ${ }_{\mathrm{E}}$ (GSK 692342)}

This is a protein adjuvant vaccine developed by GlaxoSmithKline Biologicals, USA from MTB recombinant fusion protein (Mtb32 and Mtb39) and $\mathrm{AS}_{\mathrm{B}}, \mathrm{AS}_{\mathrm{A}} \mathrm{A}$, or $\mathrm{AS} 01_{\mathrm{E}}$ adjuvant, which is designed to promote $\mathrm{BCG}$-induced immune responses [57-62]. In preclinical studies, GSK 692342 has been shown to induce more efficient immune responses in animal models than either Mtb32 or Mtb39 alone [63] as well as more efficient protection than BCG alone in mice, guinea pigs, and non-human primate $[63,64]$. In clinical phase II trials, GSK 692342 has been shown to have clinically acceptable safety and to induce strong humoral and cellular immunity in healthy and HIV-infected populations (ClinicalTrials.gov Identifier: NCT01262976), TB-infected adults (ClinicalTrials.gov Identifier: NCT01424501), and adolescents with PPD negative or positive skin reactivity (ClinicalTrials.gov Identifier: NCT00397943, NCT00621322 and NCT00950612) [58, 65-72]. At present, $\mathrm{M} 72 / \mathrm{AS} 01_{\mathrm{E}}$ has been tested in completed phase IIb clinical trial (ClinicalTrials.gov Identifier: NCT01755598) in Kenya, South Africa, and Zambia. HIVnegative LTBI adults were randomized to receive two doses of $\mathrm{M} 72 / \mathrm{AS} 01_{\mathrm{E}}$ or placebo to study the occurrence of active PTB unrelated to HIV infection and its safety or immunogenicity. Ten patients in the vaccine group were confirmed to have active PTB by bacteriology compared with 22 from the placebo group. The primary analysis of this trial showed a $54 \%$ (90\% CI: $14-75 ; P=$ 0.04 ) point estimate of vaccine efficacy over about 2 years of follow-up [73].

\section{Whole cell/extract vaccines}

\section{RUTI $^{\circledR}$}

This is an inactive, multi-antigen vaccine based on MTB whole cell extracts [74]. It is hoped that this could be used as a therapeutic vaccine in combination with short-term antituberculosis chemotherapy for prophylactic treatment of 
patients with MDR-TB. In preclinical experiments, short-term chemotherapy in mice and guinea pigs using $\mathrm{RUTI}^{\circledR}$ can effectively control LTBI, significantly induce the Th1/Th2/Th3 complex immune response, and enhance local accumulation of specific CD8 T cells [74]. Phase I studies with healthy volunteers (ClinicalTrials.gov Identifier: NCT00546273) [75] and phase II studies with LTBI (ClinicalTrials.gov Identifier: NCT01136161) [76] have found that the vaccine is safe and tolerable and has good immunogenicity at all study doses. Currently, a phase IIa clinical trial (ClinicalTrials.gov Identifier: NCT02711735) is being conducted by the University Medical Center Groningen to investigate the safety and immunogenicity of a RUTI ${ }^{\circledR}$ therapeutic vaccination for patients with MDR-TB following successful intensive-phase treatment.

\section{DAR-901 booster}

This is a whole-cell, heat-killed NTM vaccine produced from a large-scale broth growth culture method from the SRL-172 master cell bank [77]. In animal models, using DAR-901 as a heterologous booster significantly induced a strong cellular, humoral immune response in C57BL/6 mice and enhanced aerosol challenge protection against MTB when compared with BCG homologous potentiation [78]. Phase I study (ClinicalTrials.gov Identifier: NCT02063555) from the Dartmouth-Hitchcock Medical Center in the USA found that subjects (BCG-immunized adults with or without HIV) were safe and able to tolerate three doses of DAR-901 and that this effectively induced the formation of cellular and humoral immune responses [79-81]. A randomized, placebo-controlled, double-blind phase IIb clinical trial (ClinicalTrials.gov Identifier: NCT02712424) has recently been initiated with adolescents who had previously received BCG in the United Republic of Tanzania.

\section{MIP/Immuvac}

MIP, also known as Immuvac, is a heat-killed Mycobacterium indicus pranii (Mycobacterium w) vaccine. It has been approved by the drug controller general of India and the FDA as an immunotherapeutic and immunoprophylactic agent for treating multibacillary leprosy patients (as an adjunct to standard multidrug therapy), and for preventing the development of leprosy among close contacts of leprosy patients [82-84]. Mycobacterium $w$ shares an antigen with both Mycobacterium leprae as well as MTB and is also found to be useful in the prevention of TB in experimental animals $[85,86]$. Previous studies on the efficacy of Mycobacterium $w$ as an immunomodulator in PTB patients have shown higher sputum conversion rates in patients given Mycobacterium $w$ as an adjuvant therapy along with standard anti-TB treatment, and it has faster and remarkable sputum converting capacity [87].
Similar studies conducted in PTB category-II patients have shown improved cure rates [88]. As of now, the investigators are investigating MIP for its efficacy in category-II PTB (ClinicalTrials.gov Identifier: NCT00265226) and category-I PTB (ClinicalTrials.gov Identifier: NCT00341328) patients in a "double-blind placebo-controlled randomized clinical control trial."

\section{Vaccae $^{\mathrm{TM}}$}

This is a non-cell lysate of Mycobacterium vaccae, which was jointly developed by National Institutes for Food and Drug Control, China, and the 309th Hospital of the People's Liberation Army (Beijing, China) and is manufactured by Anhui Zhifei Longcom Biologic Pharmacy Co., Ltd. (Anhui, China). In 1999, Vaccae ${ }^{\mathrm{TM}}$ got the "New Drug Certificate" and approved by the National Medical Products Administration of China (NMPA) as an immunotherapeutic agent [89], which played an important key role in improving immunity, promoting phagocytosis, regulating bidirectional immunoreaction, and reducing pathological damage. It is therefore used as a combination therapy for the adjuvant treatment of TB chemotherapy and to shorten anti-tuberculosis treatment for patients with drug-sensitive TB. As a new indications for Vaccae ${ }^{\mathrm{TM}}$, NMPA approved of the plan "Phase III clinical study of efficacy and safety of Vaccae ${ }^{\mathrm{TM}}$ to prevent $\mathrm{TB}$ in high risk groups of TB infection" in December 2012. Currently, Vaccae ${ }^{\mathrm{TM}}$ was evaluated in phase III clinical trial (ClinicalTrials.gov Identifier: NCT01979900) as an LTBI immunopreventive vaccine in 2018 , and data on its safety and efficacy of TB prevention are being summarized.

\section{Attenuated/recombinant live vaccines}

\section{MTBVAC}

This is a live attenuated vaccine derived from the clinical isolate of MTB by deletion of phoP and fadD26 genes [90]. It was developed by the University of Zaragoza, Pasteur Institute, and Biofabri with support from TBVI. MTBVAC is the only TB vaccine based on attenuated MTB, which is entering clinical trials $[91,92]$. It is intended to serve as a preventive vaccine for newborns and replace BCG as well as to be a booster vaccine for adolescents and adults. However, it is likely to be subject to the same interference caused by prior immunological sensitization by NTMs as reported for BCG. Phase I clinical trial (ClinicalTrials.gov Identifier: NCT02013245) conducted in Switzerland in 2013 found that, among 18- to 45-year-old healthy adults, the MTBVAC vaccine had similar levels of safety to BCG and that no significant adverse events were caused by different doses. All vaccinated people were negative for IGRA at follow-up after 210 days of inoculation [93]. In May 2018, phase Ib clinical 
trials (ClinicalTrials.gov Identifier: NCT02729571) were completed in adults and infants. The first stage includes 18 HIV-uninfected, QFT-negative, BCG-vaccinated adult participants, randomized $1: 1$ to receive BCG Vaccine SSI or MTBVAC at equivalent dose, and the second stage was commence in 36 HIV-unexposed, BCG-naïve newborn infants, randomized 1:3 to receive BCG Vaccine SSI or MTBVAC at one of three different dose levels. Two phase Ib/IIa clinical trials (ClinicalTrials.gov Identifier: NCT03536117 and NCT02933281) and epidemiological studies (ClinicalTrials. gov Identifier: NCT03536117) have recently been carried out by Aeras and Biofabri with adults and/or infants in South Africa.

\section{BCG revaccination (Gates MRI-TBV01-201)}

BCG vaccine is given at or shortly after birth in many developing countries to prevent TB. However, it does not provide protection against PTB. For unknown reasons, a BCG revaccination or "booster," while not toxic, does not provide much additional protection. In 1995, the WHO issued the "Global Tuberculosis Program and Global Program on Vaccine: Statement on BCG Revaccination for the Prevention of Tuberculosis," which concluded that the revaccination effect of BCG could not be confirmed. WHO does not advocate to revaccinate, and it is not necessary to vaccinate anyone for multiple times [94]. After the announcement of the statement, the countries that implemented the BCG revaccination policy stopped the strategy, and China also stopped the revaccination policy of BCG in 1997. At present, only a few countries in the world still implement revaccination strategies, and some even inoculate many times. With the emergence of a large number of MDR-TB and TB-HIV co-infection at the end of the twentieth century, WHO issued another position paper on BCG in 2004 [95]. The position paper states that revaccination of BCG in individuals who are positive for TST (whether or not the positive result is caused by NTM, MTB infection, or BCG vaccination) does not improve its immunity against TB. But, if the TST is negative and inevitably close to MDR-TB, BCG may be considered. However, the 2018 [96] position paper replaces the $2004 \mathrm{WHO}$ position paper on BCG vaccine and the 2007 [97] WHO revised BCG vaccination guidelines for infants at risk for HIV infection. It incorporates recent developments in the TB field, provides revised guidance on the immunization of children infected with HIV, and reemphasizes the importance of the birth dose. The 2018 position paper shows minimal or no evidence of any additional benefit of repeat BCG vaccination against TB or leprosy. Therefore, revaccination is not recommended even if the TST reaction or result of interferon- $\gamma$ release assays (IGRA) is negative. In addition, the absence of a BCG scar after vaccination is not indicative of a lack of protection and is not an indication for revaccination. But, BCG vaccination of unvaccinated, TST-negative or IGRA-negative school children is recommended for those coming from or moving to high incidence/burden settings, as well as older groups at risk through occupational exposure.

In the mouse model, mice vaccinated with a repeat dose of BCG demonstrated an increased titer of anti-BCG IgG [98, 99], as well as revaccination of cattle with BCG 2 years after first vaccination restored protection from $\mathrm{M}$. bovis challenge compared with calves receiving only a single vaccination 2.5 years previously [100]. Interestingly, Use of an in vitro model of human PBMC induced with a repeat dose of BCG at 24 and $72 \mathrm{~h}$ of cell culture demonstrated increased antiBCG IgG levels in supernatants compared with the preboost and control groups [101]. Several human clinical studies have performed an analysis of the effects of BCG revaccination in the HIV-1-uninfected (ClinicalTrials.gov Identifier: NCT02378207 and NCT02075203), BCG-naïve healthy adults (ClinicalTrials.gov Identifier: NCT03175380), and LTBI (ClinicalTrials.gov Identifier: NCT01119521). Now, a phase IIb (ClinicalTrials.gov Identifier: NCT04152161) study of the vaccine by Aeras, in collaboration with partners including the South African Tuberculosis Vaccine Initiative (SATVI) and the Emavundleni Research Centre found that, while BCG revaccination did not demonstrate efficacy in preventing initial MTB infection (defined as QFT conversion at an IFN- $\gamma$ level of $\geq 0.35 \mathrm{IU}$ per $\mathrm{ml}$ after day 84), it did result in significantly reduced rates of sustained QFT conversion (defined as three consecutive positive QFT results after day 84) [102]. These findings have fuelled renewed interest in the potential utility of BCG revaccination and prompted consideration of BCG revaccination in certain settings as part of an overall improved TB vaccination strategy, although it is not clear to what extent cellular and/or humoral immunity contribute to this protection.

\section{VPM1002}

VPM1002 (rBCG $\Delta \mathrm{UreC}::$ hly) is a recombinant BCG vaccine developed by the Max Planck Institute for Infectious Biology, which uses the Listeriolysin O (LLO)-encoding gene of Listeria monocytogenes (Hly) to replace the urease C encoding gene (UreC) and so improve BCG immunogenicity $[93,103]$. LLO is a cholesterol-dependent cytolysin that forms a transmembrane $\beta$-barrel-like pore in the phagocytic lysosomal membrane, allowing Listeria monocytogenes to escape to the cytosol $[104,105]$. Urease inhibits Phagocytic lysosome maturation and improves the survival of MTB in macrophages $[106,107]$. Studies have found that the elimination of urease C promotes phagocytic lysosomal fusion while providing optimal $\mathrm{pH}$ for LLO stability [104]. Therefore, expression of LLO in VPM1002 leads to the release of bacterial antigens and DNA into the cytosol, ultimately triggering autophagy and apoptosis [103]. 
In preclinical studies, the VPM1002 vaccine showed superior protection to BCG in MTB aerosol challenge mice and was safe in animal models including immunodeficient mice, guinea pigs, rabbits, and non-human primates [108]. In 2009 and 2011, phase I and phase Ib clinical trials of safety and immunogenicity were carried out with adults in Germany (ClinicalTrials.gov Identifier: NCT00749034) and South Africa (ClinicalTrials.gov Identifier: NCT01113281). It was found that VPM1002 is safe and can effectively stimulate the proliferation of IFN- $\gamma$-secreting multi-factor T cells [109]. Phase II clinical trials to assess safety and immunogenicity in healthy neonates (ClinicalTrials.gov Identifier: NCT01479972) as well as HIV exposed and unexposed neonates (ClinicalTrials.gov Identifier: NCT02391415) have also been carried out in South Africa [110]. Additionally, a phase II/III trial (ClinicalTrials.gov Identifier: NCT03152903) of the vaccine for the prevention of TB recurrence in adults has been initiated in India.

\section{Evaluation of TB vaccines' efficacy}

\section{Preclinical evaluation}

Preclinical evaluations of the efficacy and safety evaluation of novel TB vaccines are the most direct means of determining whether a vaccine can enter a clinical trial. Different types of novel TB vaccines have different preclinical evaluation methods, meaning there is no unified preclinical efficacy evaluation standard for novel TB vaccines.

Researcher who are preclinically evaluating BCG typically immunize tested animals with BCG, then infect them with MTB, and finally evaluate the number of bacteria and lesion index of liver, spleen, and lung MTB as well as animal survival within a certain period. However, the types of animals used, infected strains, doses of infection, and routes of infection vary. Similar to preclinical efficacy assessment for maternal BCG, the preclinical efficacy of the recombinant BCG vaccine VPM1002 was assessed as an aerosol infection of BALB/c or C57BL6 mice by H37Rv or Beijing/W genotype family clinical isolates. Bacteriological and immunological parameters of infected mice are assessed, such as lung bacterial load, type 1 and 17 cytokine responses, $\gamma \delta \mathrm{T}$ cells, central memory cells (TCM), follicular helper T cells (TFH), and antimycobacterial antibodies [111-116]. Safety evaluations were simultaneously performed in mice (including $\mathrm{RAGl}^{-/-}$immunodeficient mice [112], severely combined immunodeficiency mice $[112,116]$ ), guinea pigs, rabbits, and non-human primates $[108,117]$ to assess rates of VPM1002 dissemination between the lungs, spleen, and other tissues as well as the ability to resist lymph node dissection $[114,116]$.

$\mathrm{M} 72 / \mathrm{AS} 01_{\mathrm{E}}$ is one of the novel TB vaccines of subunit protein adjuvant that has been highly expected in clinical trials. Its preclinical evaluation method differs from recombinant live vaccine VPM1002. Researcher of a mouse model evaluated the number of Mtb72F-specific CD8 T cells, secretion level of $\gamma$-interferon and granzyme $\mathrm{B}$, and expression abundance of CD45RB and interleukin-7 receptor $\alpha$ chain on cell surfaces in the lungs of mice after immunization with $\mathrm{M} 72 / \mathrm{AS} 01_{\mathrm{E}}$ vaccine [118]. In the guinea pig model, effects of the $\mathrm{M} 72 / \mathrm{AS} 01_{\mathrm{E}}$ vaccine on survival rate, $\mathrm{PTB}$ granuloma, and airway remodeling and reconstruction were evaluated [119]. The rabbit model of tuberculous meningitis evaluates the protective effect of the M72/AS01 ${ }_{\mathrm{E}}$ vaccine on the central nervous system through clearance rates of TB in cerebrospinal fluid, leukocyte level in cerebrospinal fluid, and pathological damage in brain and lung, as well as changes in body weight [120]. For the cynomolgus monkey, the clinical parameters, pathology, long- and short-term survival rates, Th1 cytokines (IFN- $\gamma$, TNF, IL-2, and so on), and innate cytokine IL- 6 changes were mainly evaluated [121].

However, there is a consensus that the international evaluation of preclinical efficacy and safety of novel TB vaccines using mouse, guinea pig/rabbit, and non-human primate threelevel evaluation models is consistent, although evaluation of different animal types has its advantages and limitations (Table 2).

\section{Clinical evaluation}

Clinical trials are the only way to research and develop vaccines. Vaccines that have been preclinically evaluated need to undergo phase I, II, and III clinical trials before obtaining registration approval. It may even be necessary to design phase IV clinical trials to comprehensively evaluate the safety and efficacy of post-marketing vaccines in practical populations. The clinical evaluation of novel TB vaccines is based on TB incidence in phase III clinical results. Vaccine efficacy is evaluated by comparing the difference in TB incidence between the vaccine and placebo groups. The WHO required novel TB vaccines to have a protective power of at least $50 \%$. Phase I and II clinical trials are a relatively simple means of evaluating the safety and immunogenicity of novel TB vaccines. However, due to long-term and delayed recurrence of MTB infection, efficacy evaluation of TB vaccines in phase III clinical trials typically requires a follow-up of at least 25 years for large samples.

The lack of intertrial harmonization or standardization for the clinical evaluation of different novel TB vaccine candidates (Table 3) precludes a direct comparison of those immunological outcomes. Researcher of phase I and phase II clinical studies of vaccines for neonates or uninfected individuals have tended to find that vaccine groups can induce cytokine production as well as enhancing anti-TB immune response. However, when clinically evaluating the LTBI adult prophylactic vaccine, immunized subjects were infected with MTB, 
Table 2 Advantages and disadvantages of animal models in preclinical evaluation of TB vaccine

\begin{tabular}{|c|c|c|}
\hline $\begin{array}{l}\text { Animal } \\
\text { model }\end{array}$ & Advantages & Disadvantages \\
\hline Mouse & $\begin{array}{l}\text { - Small size, easy to operate and low purchase and feeding } \\
\text { costs [122]. } \\
\text { - Rich in immunoassay reagents and has a clear genetic } \\
\text { background and can be used to study the pathogenesis of TB } \\
\text { [123, 124]. } \\
\text { - Humanized mouse model can be constructed for the study of } \\
\text { TB-HIV co-infection [125]. }\end{array}$ & $\begin{array}{l}\text { - Without obvious clinical manifestation of TB infection [126]. } \\
\text { - Produces a weak late-type hypersensitivity reaction and induces TB } \\
\text { granulomas, but the structure of TB granulomas is different from that } \\
\text { of humans [127, 128]. } \\
\text { - The sensitivity to MTB is low, and the pathological changes and } \\
\text { bacterial load in the organs are uneven [129]. }\end{array}$ \\
\hline Guinea pig & $\begin{array}{l}\text { - Susceptibility to MTB and low dose infection can cause } \\
\text { significant TB lesions [130]. } \\
\text { - Producing a strong delayed type hypersensitivity response } \\
\text { and inducing caseous necrosis [130]. } \\
\text { - Miliary nodules are observed in the lungs, liver, and spleen, } \\
\text { and TB granulomas are very similar to humans [130, 131]. } \\
\text { - Anti-TB drugs and vaccines respond well and are commonly } \\
\text { used models for TB skin test assays [132]. }\end{array}$ & $\begin{array}{l}\text { - Lack of general clinical manifestations of TB infection [132]. } \\
\text { - Limited availability of immune reagents }[130,132] \text {. } \\
\text { - Easy to form a wide range of diffuse lesions and cannot spontaneously } \\
\text { latent infection [130]. }\end{array}$ \\
\hline Rabbit & $\begin{array}{l}\text { - TB granulomas with caseous necrosis and liquefaction, and } \\
\text { easy to form cavitation [133]. } \\
\text { - Pathological model of bone TB, TB meningitis, and skin TB } \\
\text { liquefaction can be constructed [134-139]. }\end{array}$ & $\begin{array}{l}\text { - Higher purchase and feeding costs [133]. } \\
\text { - Limited availability of immune reagents [133]. } \\
\text { - Easy to form haematogenous spread and death [133]. }\end{array}$ \\
\hline $\begin{array}{l}\text { Non-human } \\
\text { primate }\end{array}$ & $\begin{array}{l}\text { - General clinical manifestations of TB are consistent with } \\
\text { humans (low fever, emaciation, cough, and dyspnea) } \\
\text { [140-143]. } \\
\text { - Can mimic LTBI and various forms of active TB [144, 145]. } \\
\text { - Structure of TB granuloma is similar to human [142]. }\end{array}$ & $\begin{array}{l}\text { - Higher purchase and feeding costs and containment facilities [142]. } \\
\text { - Limited availability of immune reagents [142]. } \\
\text { - High variation within groups, making it difficult to evaluate the } \\
\text { effectiveness of vaccines [142]. }\end{array}$ \\
\hline
\end{tabular}

and sensitization states and immune markers of the body were found to differ from those who were uninfected. A series of immune indicators commonly used to evaluate vaccines for neonates or uninfected individuals have been positive prior to vaccination. Whether changes in these indicators following vaccination can be applied to the clinical evaluation of LTBI anti-vaccination vaccines remains unknown. Therefore, it is even more rare if a standard set of parameters allows accurate comparisons between studies and vaccines to determine whether this is a reproducible in clinical trials. At present, novel vaccines for TB in phase III clinical trials are MIP/ Immuvac, Vaccae ${ }^{\mathrm{TM}}$, and VPM1002, and no relevant research data have been published [146, 147].

\section{Challenges of researching TB vaccines}

The ideal TB vaccine will have a strong protective efficacy, stable and long-lasting immunogenicity, and no adverse reactions. It would prevent TB by single or several immunizations. However, there is no vaccine that is more protective or sustainable than BCG. While some novel vaccine candidates have achieved certain effects in animal models, how to apply them to humans and whether they can replace BCG still requires a long period of experimentation, follow-up, and demonstration. MVA85A is the first new TB vaccine to receive much attention in the past 90 years. In preclinical animal models, MVA85A was found to significantly enhance BCG immunogenicity priming in guinea pigs, rhesus monkeys, and cattle [148]. Its safety and tolerability have also been validated in phase I and phase IIa clinical trials in healthy adults and TBand HIV-infected infants, children, and adolescents [40, 149-156]. However, in a phase IIb clinical trial conducted in South Africa in 2013, it was found that healthy infants who had been vaccinated with BCG and immunized with MVA85A did not have additional protection [157]. This result was a great disappointment to both the TB vaccine research community and funders and a call to action for researchers in basic and applied vaccine development. At the same time, this lesson suggests that some of the results obtained in preclinical animal models do not align with results of clinical trials. Therefore, the development of novel TB vaccines continues to face many challenges.

(1) Lack of clear protective antigens: MTB has approximately 4000 genes encoding thousands of proteins [158]. The proteins used in TB vaccine design are mainly proliferation-associated antigens (that is, six of the eight subunit vaccines entering clinical studies contain Ag85A or Ag85B proteins). Critical and unresolved questions in TB vaccinology are how to select the best antigens and how many antigen to include in a vaccine. However, limited clinical trial data makes it difficult to support the effectiveness of this antigen or any other single antigen against TB. It is therefore necessary to select more effective antigens to construct a vaccine from the 


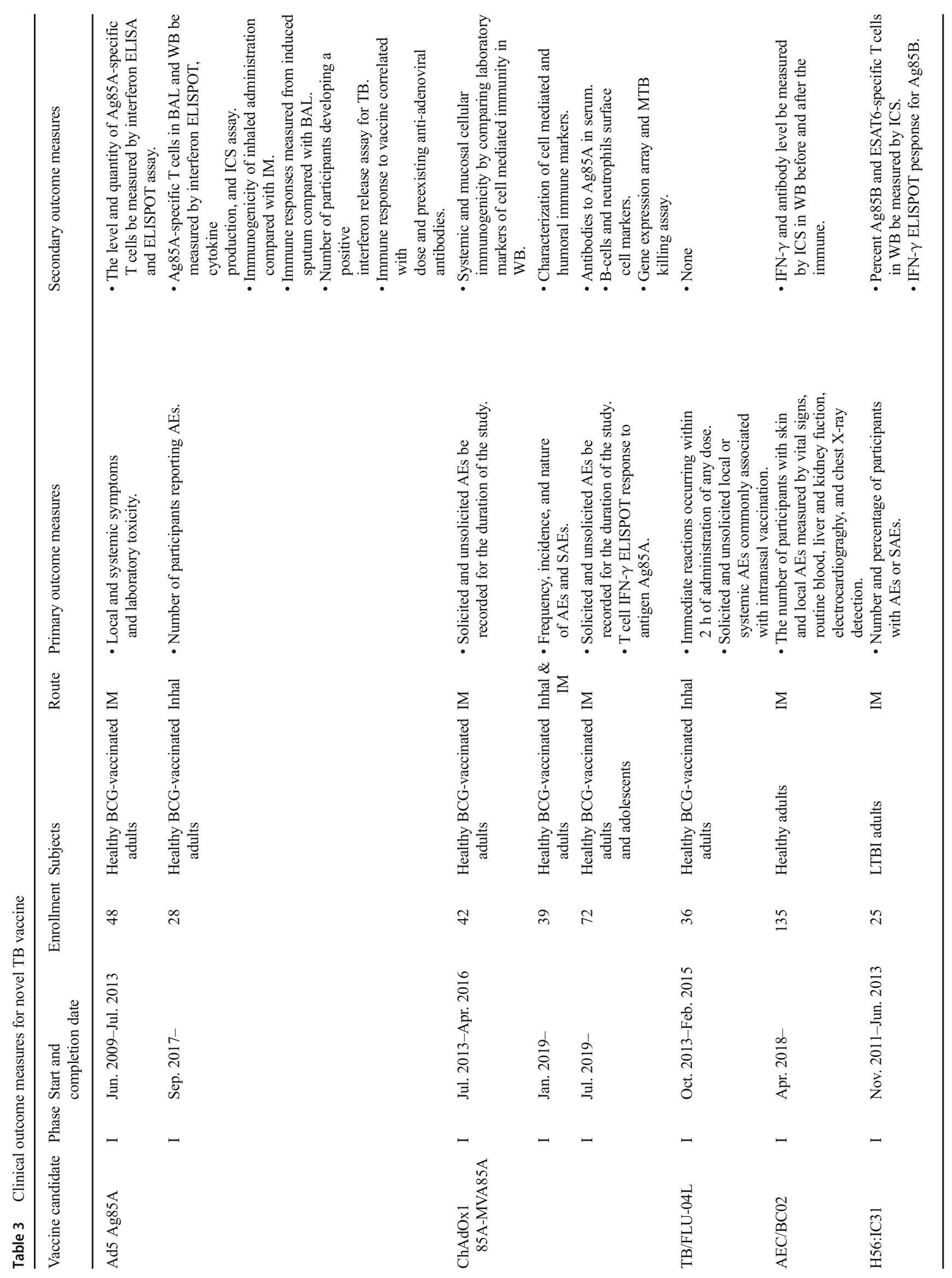




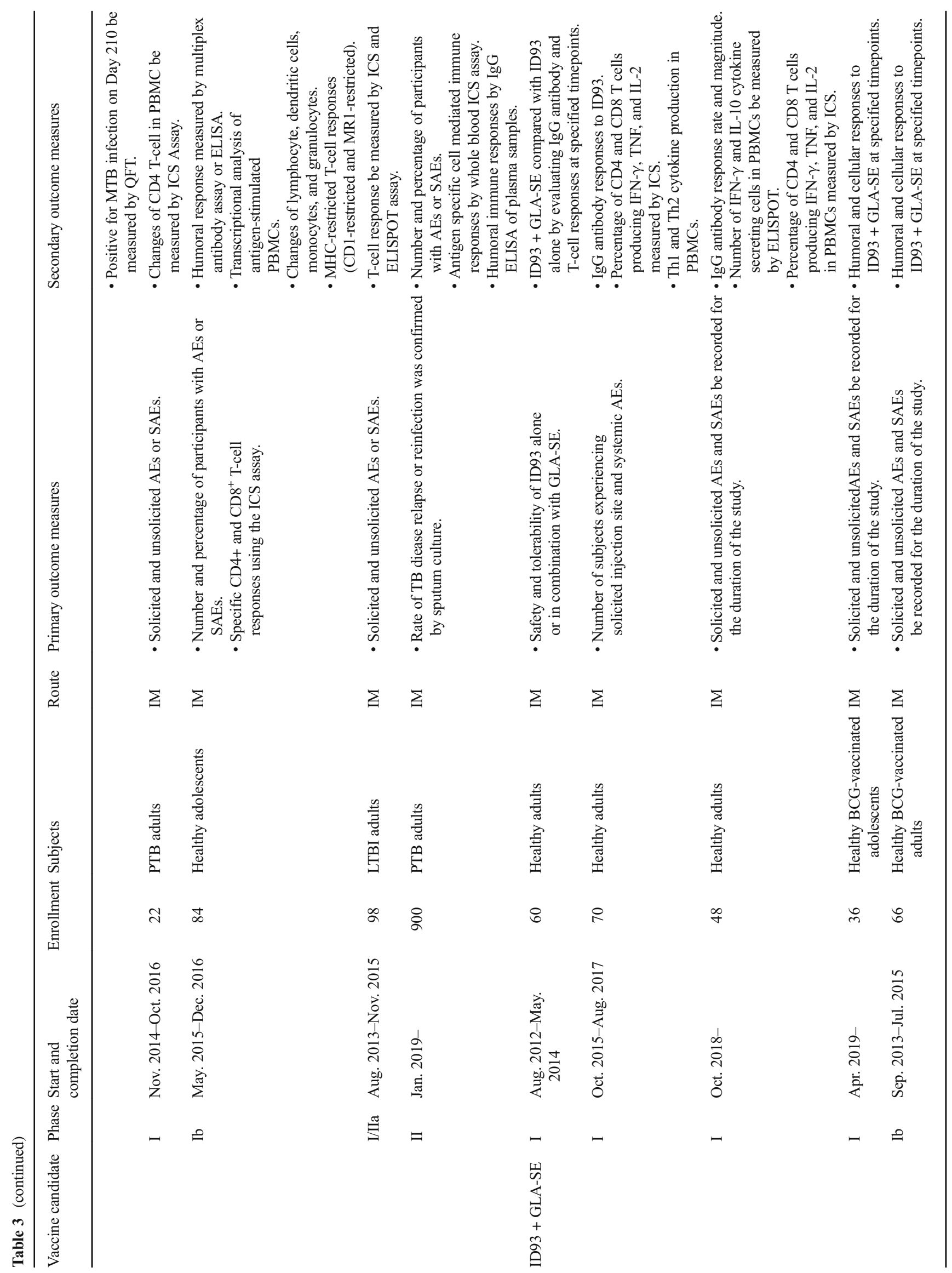




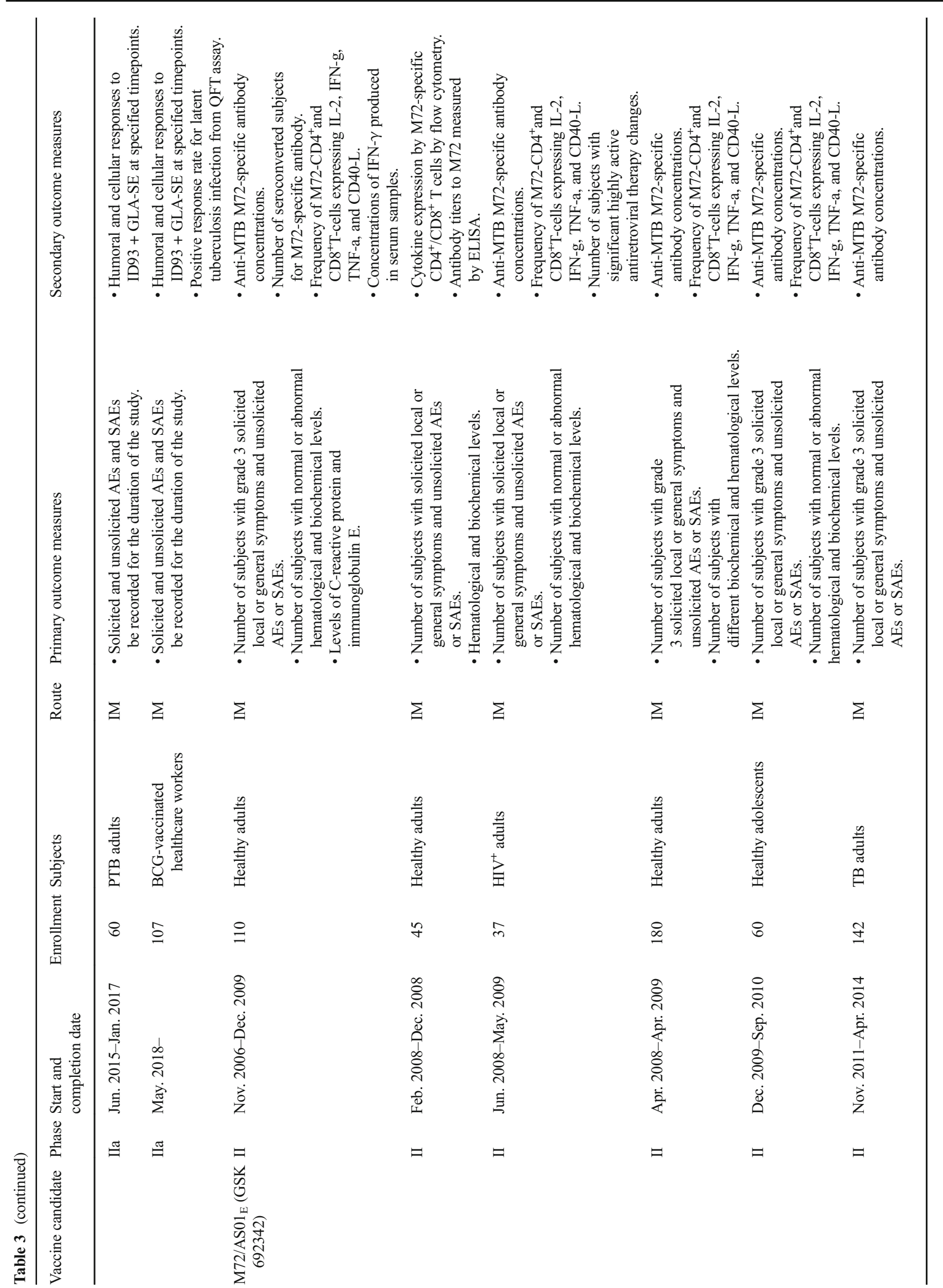



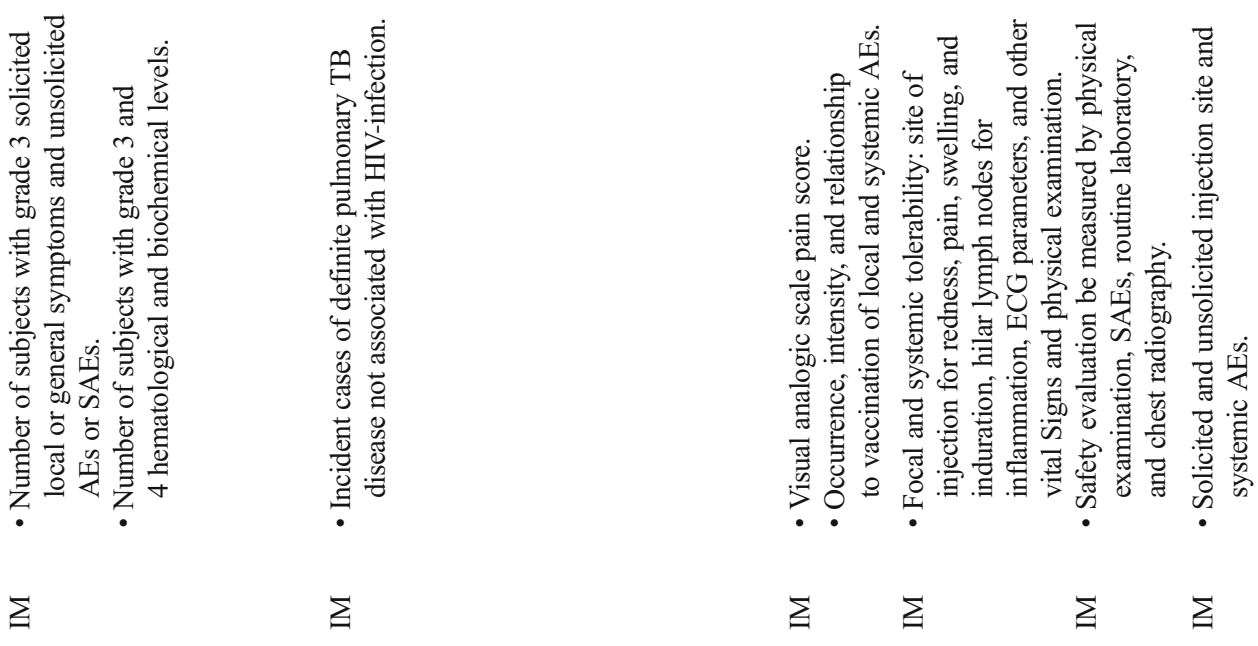

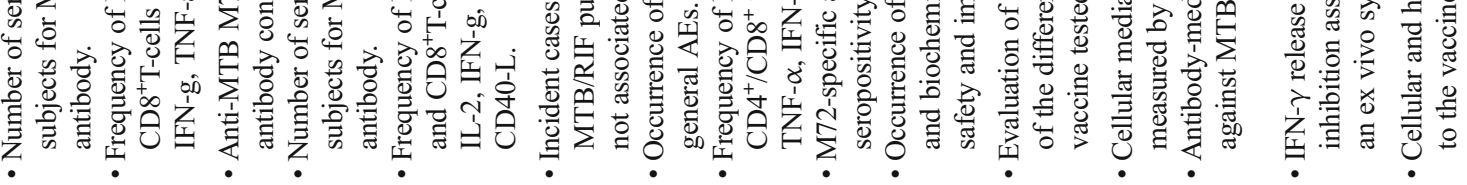

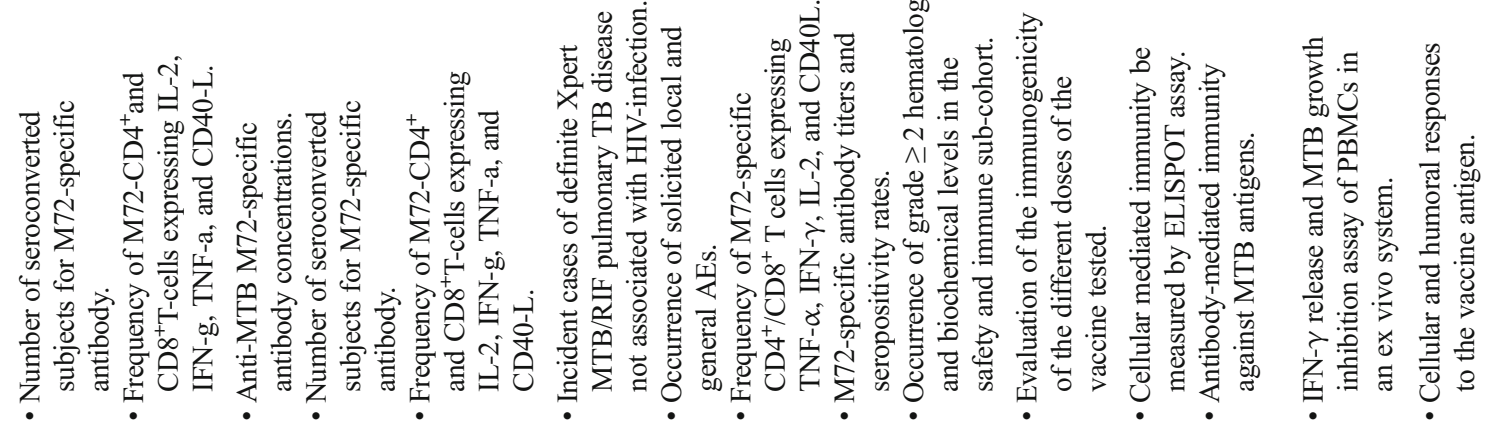

$\stackrel{n}{\stackrel{2}{*}}$

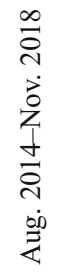

d

ก

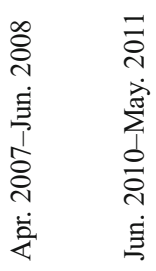

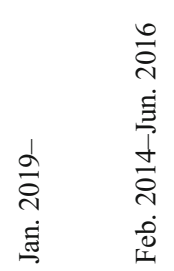

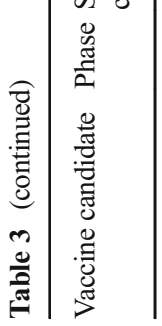

$=$

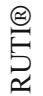

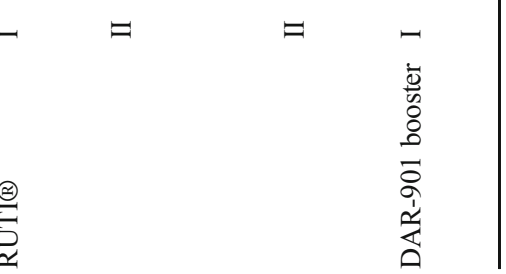



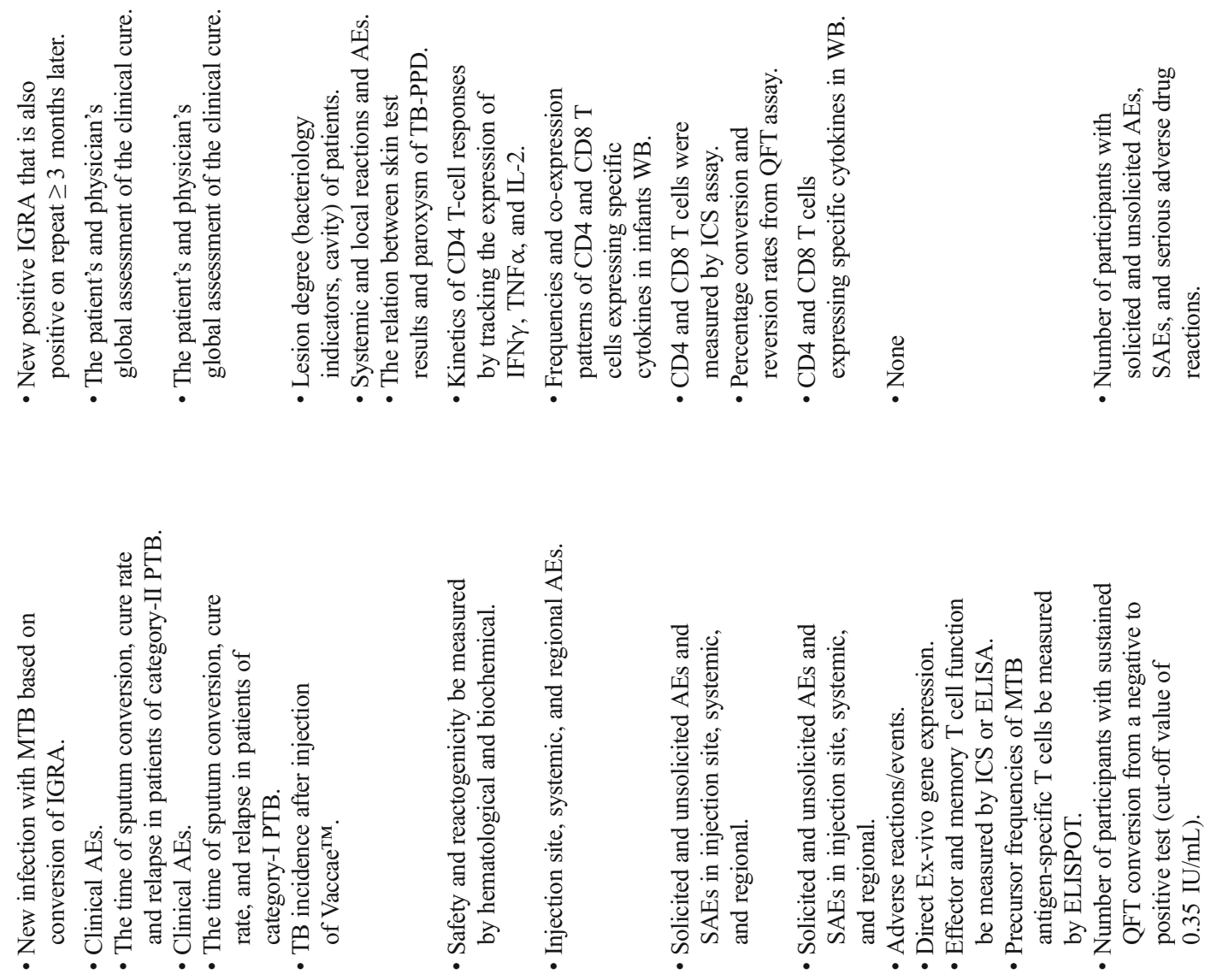

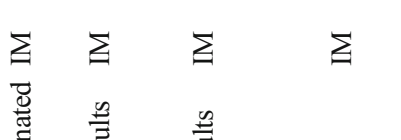

$\ominus$

○

$\cong$

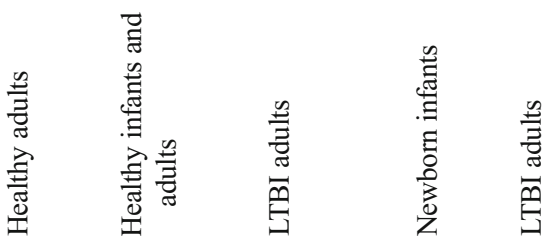

目

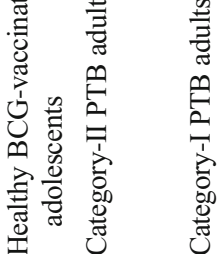

兽

疋

品

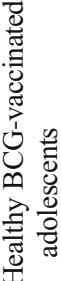

గี ๕

in

ิ

a $\infty$

$\stackrel{8}{\stackrel{8}{-1}}$

离

完

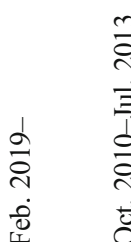

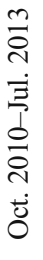

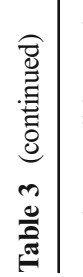

고 ฮั

กิธ

离

ज्ञ
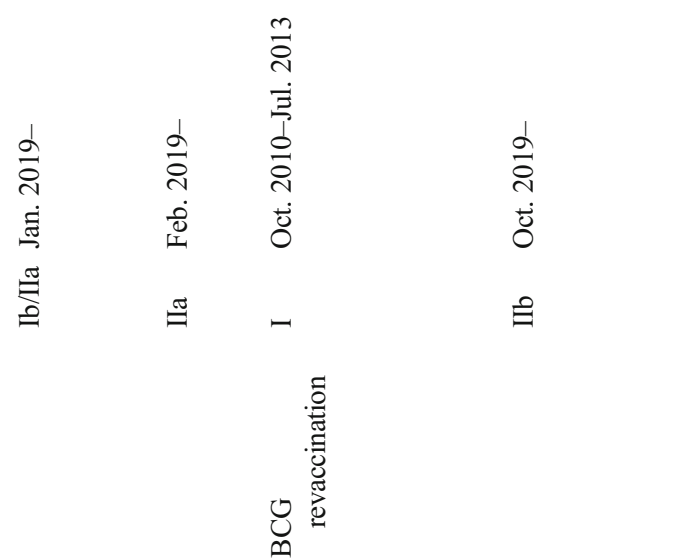


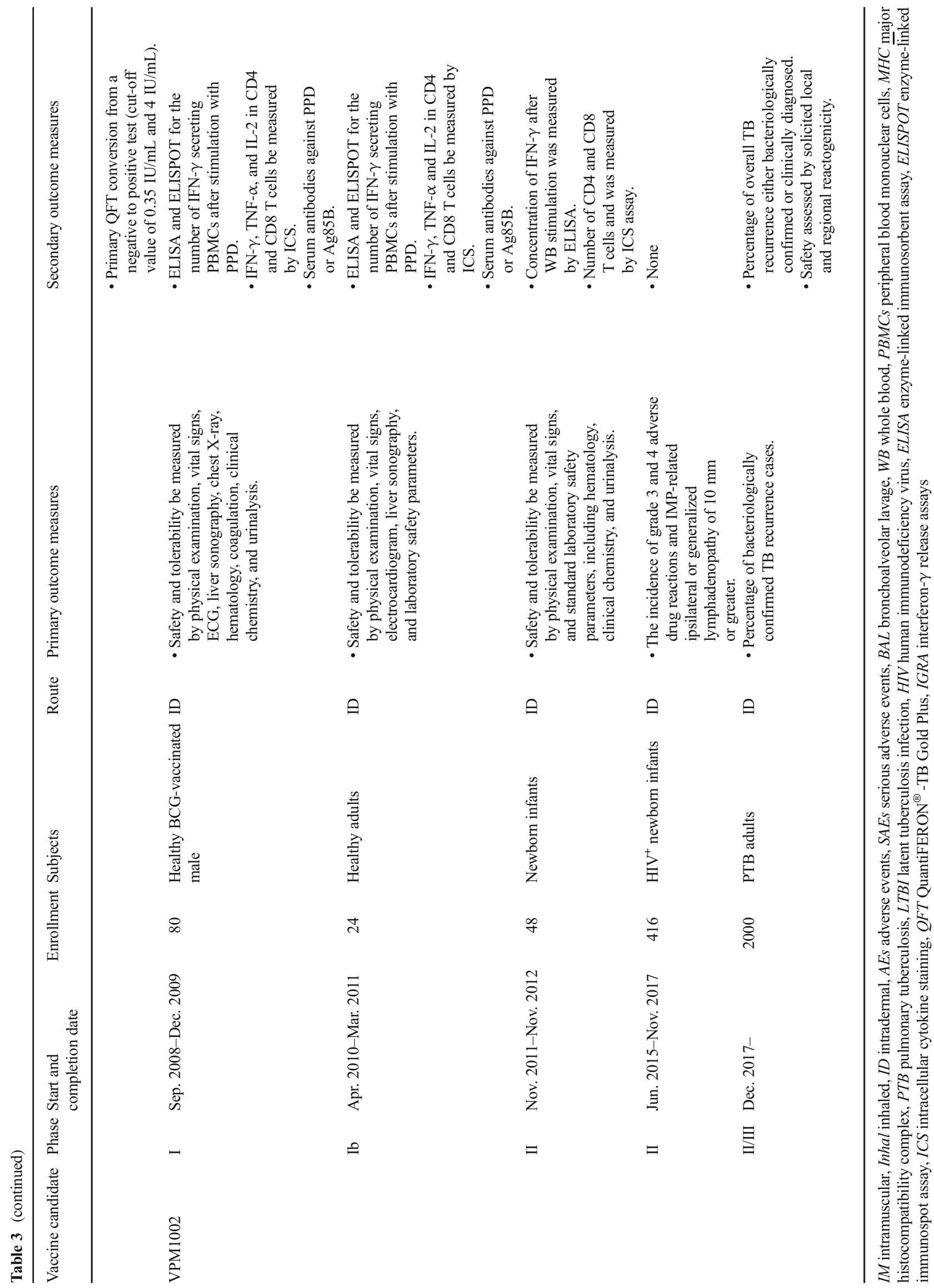


mechanism of MTB infection and so induce more effective protective immunity [159].

(2) Lack of credible preclinical evaluation indicators: In early evaluation of immunogenicity, the novel TB vaccine mainly detects specific antibody titers induced by the vaccine, different types of cytokines produced by $\mathrm{CD}^{+}$ and $\mathrm{CD}^{+}$, and other $\mathrm{T}$ cells following immunization [160]. However, the correlation between these specific immune cells or cytokines in the host's immune protection against natural TB infection needs further study [161].

(3) Lack of suitable vaccine evaluation animal model: Animal experiments have provided evaluation of vaccines in terms of immunogenicity, safety, and efficacy. Mouse models have the advantages of low price, strong viability, and easy access to detection reagents, meaning they are the first choice for the evaluating the preliminary effects of novel vaccines [162]. However, mouse models do not accurately reflect the authenticity of vaccine protection, especially when studying the longacting immune protection response. Therefore, the use of mouse models to evaluate the effect of novel TB vaccines may result in a deviation from the actual clinical application effect [163]. In addition, evaluation results from the same vaccines in different animal models were inconsistent, with mice and guinea pigs achieving good immunoprotective effects, which failed in non-human primates [164]. Infection and disease manifestations of MTB vary from species to species, and immune response following vaccination is more variable in humans [23]. Therefore, differences between humans and animals may be one of the main factors affecting the predictive ability of animal models.

(4) Lack of emulated exposure methods: A significant difference exists in the nature of exposure between animal models and natural human infections. In preclinical animal models, MTB are initially infected by high-dose, single-strain, single exposure through various routes of attack (such as aerosol, tail vein, or intraperitoneal injection). However, in natural conditions, humans often experience multiple low doses, and infections are established following exposure to different strains [165].

(5) Lack of a unified clinical trial endpoint criteria: Reducing the incidence of TB and improving the disease status of patients are the main criteria for evaluating TB vaccines. In preclinical animal models, the effectiveness of vaccines tends to be assessed by improvements in organ bacterial counts, histopathological damage, and time of animal death. In contrast, scientists use TB incidence as an end point of human clinical trials. Obviously, there is a fundamental difference and lack of relevance between preclinical trials and clinical trials in determining the endpoint of vaccine effectiveness.
(6) Lack of consistent evaluation environment: Unlike in a single laboratory environment, there may be multiple uncertainties in a human clinical test environment, such as diet, nutritional status, NTM, worm co-infection, and host genetic heterogeneity on susceptibility to TB. In contrast, preclinical animal models tend to use specific pathogen-free animals with a consistent genetic background.

(7) Immunization population is complicated: When compared with other infectious disease vaccines, a difficulty in researching novel TB vaccines is that the immune status of the vaccination population or infection background is complicated. The widespread vaccination of BCG and NTM infections in countries that have high rates of TB further exacerbates the complexity of vaccination populations. In particular, whether a vaccination with a strong immunogenic vaccine in the LTBI population leads to a Koch response is a safety issue that cannot be ignored. Therefore, those who are BCG naïve, negative, or maintain positive after BCG vaccination and LTBI need to be accurately differentiated and accurately immunized to optimize vaccination effectiveness.

\section{The future of TB vaccine research}

Since the first use of BCG, there have been controversies about its advantages and disadvantages, including its safety, sensitivity to the diagnostic reagent tuberculin, and especially the failure of some BCG vaccination trials. Novel TB vaccines, which provide effective, sustained protection, have always been an international priority. Especially in recent years, given the advantages of novel vaccine research technology, new methods and ideas for various vaccine research have been established. A large number of different vaccine candidates and their advanced stages in clinical development denote a unique and exciting phase in TB vaccine research. There are also a large number of novel vaccine candidates in preclinical development, including more recently developed vaccine formats such as DNA vaccines, new adjuvants and delivery systems, and combination vaccines. In summary, the development of novel TB vaccines remains mostly focused on the following regimens:

(1) Priming vaccine: Developing a new vaccine with longer lasting immunoprotective properties and better protection than BCG to replace the primary vaccination of BCG.

(2) Booster vaccine following BCG vaccination: Performing short- or long-term immunization and heterologous or homologous booster to enhance or prolong BCG priming protective effects, improving immunogenicity of BCG, 
and making up for the shortcomings of BCG long-term effects.

(3) Latent infection preventive vaccine: Used to inhibit endogenous re-ignition of infected individuals with MTB while preventing exogenous re-infection.

(4) Therapeutic vaccine: As an effective supplement to conventional chemotherapy, which can reduce lesions, promote sputum MTB negative and cavity closure, shorten the course of treatment, consolidate the effect of chemotherapy, and promote the outcome of the disease, effectively treating drug-resistant TB.

\section{Conclusion}

TB remains one of the greatest threats to global health, meaning the importance of effective and safer vaccines for controlling this epidemic is undisputed, particularly for MDR-TB and TB-HIV co-infection. In the past 30 years, TB vaccines have developed greatly, and vaccine candidates for different age groups and different forms of TB have emerged. Despite disappointing results from some of the clinical efficacy trials, these experiences are sufficient to remind us to revisit the design and evaluation of TB vaccines. Today, we are witnessing immense progress in both preclinical and clinical TB vaccine research. With the continuous coordination and cooperation of the TB vaccine community, building a world free of TB may be just around the corner.

Author contribution Junli Li, Chuan Qin, and Lingjun Zhan conceived the project direction; Junli Li, Jun Tang, and Yanan Shi researched and collated all the relevant literature; Aihua Zhao and Guozhi Wang revised and edited the manuscript; Junli Li wrote the manuscript.

\section{Compliance with ethical standards}

Conflict of interest The authors declare that they have no conflict of interest.

Funding information This work was supported by the National Science and Technology Major Project for Infectious Diseases Control and Prevention (No.2017ZX10201301-006) and CAMS Major Innovation Funding For Medical Science (2016-I2M-2-006).

\section{References}

1. Correa-Macedo W et al (2019) The interplay of human and Mycobacterium tuberculosis Genomic Variability. Front Genet 18(10):865

2. Mustafa AS (2005) Mycobacterial gene cloning and expression, comparative genomics, bioinformatics and proteomics in relation to the development of new vaccines and diagnostic reagents. Med Princ Pract 14(Suppl 1):27-34
3. Hershkovitz I et al (2015) Tuberculosis origin: the Neolithic scenario. Tuberculosis (Edinb) 95(Suppl 1):S122-S126

4. World Health Organization (2019) Global tuberculosis report 2019. WHO, Geneva

5. World Health Organization (1994) TB: a global emergency, WHO report on the TB epidemic (WHO/TB/94.177). WHO, Geneva

6. Styblo K, Meijer J, Sutherland I (1969) The transmission of tubercle bacilli: its trend in a human population. Bull World Health Organ 41(1):137-178

7. D'Arcy Hart P (2001) Historical declines in tuberculosis: nature, nurture and the biosocial model. Int J Tuberc Lung Dis 5(9):879

8. Pai M et al (2016) Tuberculosis. Nat Rev Dis Primers 2:16076

9. Schorey JS, Schlesinger LS., Innate Immune Responses to Tuberculosis. Microbiol Spectr, 2016 Dec; 4(6). https://doi.org/ 10.1128/microbiolspec.TBTB2-0010-2016

10. DeWeerdt S (2013) Vaccines: an age-old problem. Nature 502(7470):S8-S9

11. Calmette A (1931) Preventive vaccination against tuberculosis with BCG. Proc R Soc Med 24(11):1481-1490

12. Brosch $\mathrm{R}$ et al (2007) Genome plasticity of BCG and impact on vaccine efficacy. Proc Natl Acad Sci U S A 104(13):5596-5601

13. Favorov $\mathrm{M}$ et al (2012) Comparative tuberculosis (TB) prevention effectiveness in children of Bacillus Calmette-Guerin (BCG) vaccines from different sources Kazakhstan. PLoS One 7(3):e32567

14. Mostowy $\mathrm{S}$ et al (2003) The in vitro evolution of BCG vaccines. Vaccine 21(27-30):4270-4274

15. Trunz BB, Fine P, Dye C (2006) Effect of BCG vaccination on childhood tuberculous meningitis and miliary tuberculosis worldwide: a meta-analysis and assessment of cost-effectiveness. Lancet 367(9517):1173-1180

16. Blok BA et al (2015) Trained innate immunity as underlying mechanism for the long-term, nonspecific effects of vaccines. $\mathrm{J}$ Leukoc Biol 98(3):347-356

17. Roth A et al (2006) Bacillus Calmette-Guerin vaccination and infant mortality. Expert Rev Vaccines 5(2):277-293

18. Andersen P, Kaufmann SH., Novel vaccination strategies against tuberculosis.Cold Spring Harb Perspect Med. 2014 Jun 2;4(6). pii: a018523. https://doi.org/10.1101/cshperspect.a018523

19. Rodrigues LC, Mangtani P, Abubakar I (2011) How does the level of BCG vaccine protection against tuberculosis fall over time? BMJ 343:d5974

20. Mangtani $P$ et al (2014) Protection by BCG vaccine against tuberculosis: a systematic review of randomized controlled trials. Clin Infect Dis 58(4):470-480

21. Abubakar I et al (2013) Systematic review and meta-analysis of the current evidence on the duration of protection by bacillus Calmette-Guerin vaccination against tuberculosis. Health Technol Assess 17(37):1-372 v-vi

22. Andersen P, Doherty TM (2005) The success and failure of BCG implications for a novel tuberculosis vaccine. Nat Rev Microbiol 3(8):656-662

23. Colditz GA et al (1994) Efficacy of BCG vaccine in the prevention of tuberculosis. Meta-analysis of the published literature. JAMA 271(9):698-702

24. Fine PE (1995) Variation in protection by BCG: implications of and for heterologous immunity. Lancet 346(8986):1339-1345

25. Colditz GA et al (1995) The efficacy of bacillus Calmette-Guerin vaccination of newborns and infants in the prevention of tuberculosis: meta-analyses of the published literature. Pediatrics 96(1 Pt 1):29-35

26. Gallant CJ et al (2010) Impact of age and sex on mycobacterial immunity in an area of high tuberculosis incidence. Int J Tuberc Lung Dis 14(8):952-959

27. Trial of BCG vaccines in south India for tuberculosis prevention (1979) first report-Tuberculosis Prevention Trial. Bull World Health Organ 57(5):819-827 
28. Bulletin of the World Health Organization (1979) Trial of BCG vaccines in south India for tuberculosis prevention: first report. WHO, Geneva

29. Harris DP et al (2005) Regulation of IFN-gamma production by B effector 1 cells: essential roles for T-bet and the IFN-gamma receptor. J Immunol 174(11):6781-6790

30. Wagner $M$ et al (2004) IL-12p70-dependent Th1 induction by human B cells requires combined activation with CD40 ligand and CpG DNA. J Immunol 172(2):954-963

31. Wang J et al (2004) Single mucosal, but not parenteral, immunization with recombinant adenoviral-based vaccine provides potent protection from pulmonary tuberculosis. J Immunol 173(10): $6357-6365$

32. Vordermeier HM et al (2009) Viral booster vaccines improve Mycobacterium bovis BCG-induced protection against bovine tuberculosis. Infect Immun 77(8):3364-3373

33. Dean $\mathrm{G}$ et al (2014) Comparison of the immunogenicity and protection against bovine tuberculosis following immunization by BCG-priming and boosting with adenovirus or protein based vaccines. Vaccine 32(11):1304-1310

34. Metcalfe HJ et al (2018) Ag85A-specific CD4 ${ }^{+} \mathrm{T}$ cell lines derived after boosting BCG-vaccinated cattle with Ad5-85A possess both mycobacterial growth inhibition and anti-inflammatory properties. Vaccine 36(20):2850-2854

35. Santosuosso $M$ et al (2006) Intranasal boosting with an adenovirus-vectored vaccine markedly enhances protection by parenteral Mycobacterium bovis BCG immunization against pulmonary tuberculosis. Infect Immun 74(8):4634-4643

36. Smaill F, Xing Z (2014) Human type 5 adenovirus-based tuberculosis vaccine: is the respiratory route of delivery the future? Expert Rev Vaccines 13(8):927-930

37. Smaill $\mathrm{F}$ et al (2013) A human type 5 adenovirus-based tuberculosis vaccine induces robust $T$ cell responses in humans despite preexisting anti-adenovirus immunity. Sci Transl Med 5(205): 205 ra134

38. Jeyanathan $\mathrm{M}$ et al (2016) Induction of an immune-protective Tcell repertoire with diverse genetic coverage by a novel viralvectored tuberculosis vaccine in humans. J Infect Dis 214(12): 1996-2005

39. Stylianou E et al (2015) Improvement of BCG protective efficacy with a novel chimpanzee adenovirus and a modified vaccinia Ankara virus both expressing Ag85A. Vaccine 33(48):6800-6808

40. Hawkridge $T$ et al (2008) Safety and immunogenicity of a new tuberculosis vaccine, MVA85A, in healthy adults in South Africa. J Infect Dis 198(4):544-552

41. Dockrell HM (2016) Towards new TB vaccines: what are the challenges? Pathog Dis 74(4):ftw016

42. Lu JB et al (2016) Analysis of Koch phenomenon of Mycobacterium tuberculosis-infected guinea pigs vaccinated with recombinant tuberculosis vaccine $\mathrm{AEC} / \mathrm{BC} 02$. Zhonghua Jie $\mathrm{He}$ He Hu Xi Za Zhi 39(7):524-528

43. Perez-Martinez AP et al (2017) Conservation in gene encoding Mycobacterium tuberculosis antigen Rv2660 and a high predicted population coverage of H56 multistage vaccine in South Africa. Infect Genet Evol 55:244-250

44. Lin PL et al (2012) The multistage vaccine H56 boosts the effects of BCG to protect cynomolgus macaques against active tuberculosis and reactivation of latent Mycobacterium tuberculosis infection. J Clin Invest 122(1):303-314

45. Suliman $\mathrm{S}$ et al (2019) Dose optimization of H56:IC31 vaccine for tuberculosis-endemic populations. A double-blind, placebo-controlled, dose-selection trial. Am J Respir Crit Care Med 199(2): 220-231

46. Luabeya AK et al (2015) First-in-human trial of the post-exposure tuberculosis vaccine H56:IC31 in Mycobacterium tuberculosis infected and non-infected healthy adults. Vaccine 33(33):4130 4140

47. Orr MT et al (2014) A dual TLR agonist adjuvant enhances the immunogenicity and protective efficacy of the tuberculosis vaccine antigen ID93. PLoS One 9(1):e83884

48. Duthie MS et al (2014) Protection against Mycobacterium leprae infection by the ID83/GLA-SE and ID93/GLA-SE vaccines developed for tuberculosis. Infect Immun 82(9):3979-3985

49. Bertholet $\mathrm{S}$ et al (2010) A defined tuberculosis vaccine candidate boosts BCG and protects against multidrug-resistant Mycobacterium tuberculosis. Sci Transl Med 2(53):53ra74

50. Baldwin SL et al (2012) The importance of adjuvant formulation in the development of a tuberculosis vaccine. J Immunol 188(5): 2189-2197

51. Baldwin SL et al (2016) Protection and long-lived immunity induced by the ID93/GLA-SE vaccine candidate against a clinical Mycobacterium tuberculosis isolate. Clin Vaccine Immunol 23(2): 137-147

52. Cha SB et al (2016) Pulmonary immunity and durable protection induced by the ID93/GLA-SE vaccine candidate against the hyper-virulent Korean Beijing Mycobacterium tuberculosis strain K. Vaccine 34(19):2179-2187

53. Coler RN et al (2013) Therapeutic immunization against Mycobacterium tuberculosis is an effective adjunct to antibiotic treatment. J Infect Dis 207(8):1242-1252

54. Baldwin SL et al (2014) The ID93 tuberculosis vaccine candidate does not induce sensitivity to purified protein derivative. Clin Vaccine Immunol 21(9):1309-1313

55. Coler RN et al (2018) The TLR-4 agonist adjuvant, GLA-SE, improves magnitude and quality of immune responses elicited by the ID93 tuberculosis vaccine: first-in-human trial. NPJ Vaccines 3:34

56. Penn-Nicholson A et al (2018) Safety and immunogenicity of the novel tuberculosis vaccine ID93 + GLA-SE in BCG-vaccinated healthy adults in South Africa: a randomised, double-blind, placebo-controlled phase 1 trial. Lancet Respir Med 6(4):287-298

57. Homolka S, Ubben T, Niemann S (2016) High sequence variability of the ppE18 gene of clinical Mycobacterium tuberculosis complex strains potentially impacts effectivity of vaccine candidate M72/AS01E. PLoS One 11(3): 0152200

58. Montoya J et al (2013) A randomized, controlled dose-finding phase II study of the M72/AS01 candidate tuberculosis vaccine in healthy PPD-positive adults. J Clin Immunol 33(8):1360-1375

59. Skeiky YA et al (1999) Cloning, expression, and immunological evaluation of two putative secreted serine protease antigens of Mycobacterium tuberculosis. Infect Immun 67(8):3998-4007

60. Dillon DC et al (1999) Molecular characterization and human Tcell responses to a member of a novel Mycobacterium tuberculosis mtb39 gene family. Infect Immun 67(6):2941-2950

61. Al-Attiyah R et al (2004) In vitro cellular immune responses to complex and newly defined recombinant antigens of Mycobacterium tuberculosis. Clin Exp Immunol 138(1):139-144

62. Montagnani $\mathrm{C}$ et al (2014) Vaccine against tuberculosis: what's new? BMC Infect Dis 14(Suppl 1):S2

63. Nabavinia MS et al (2012) Construction of an expression vector containing Mtb72F of Mycobacterium tuberculosis. Cell J 14(1): 61-66

64. Skeiky YA et al (2004) Differential immune responses and protective efficacy induced by components of a tuberculosis polyprotein vaccine, Mtb72F, delivered as naked DNA or recombinant protein. J Immunol 172(12):7618-7628

65. Day CL et al (2013) Induction and regulation of T-cell immunity by the novel tuberculosis vaccine M72/AS01 in south African adults. Am J Respir Crit Care Med 188(4):492-502 
66. Gillard P et al (2016) Safety and immunogenicity of the M72/ ASO1E candidate tuberculosis vaccine in adults with tuberculosis: a phase II randomised study. Tuberculosis (Edinb) 100:118-127

67. Idoko OT et al (2014) Safety and immunogenicity of the M72/ AS01 candidate tuberculosis vaccine when given as a booster to BCG in Gambian infants: an open-label randomized controlled trial. Tuberculosis (Edinb) 94(6):564-578

68. Kumarasamy $\mathrm{N}$ et al (2016) A randomized, controlled safety, and immunogenicity trial of the M72/AS01 candidate tuberculosis vaccine in HIV-positive Indian adults. Medicine (Baltimore) 95(3):e2459

69. Leroux-Roels I et al (2013) Improved CD4(+) T cell responses to Mycobacterium tuberculosis in PPD-negative adults by M72/ AS01 as compared to the M72/AS02 and Mtb72F/AS02 tuberculosis candidate vaccine formulations: a randomized trial. Vaccine 31(17):2196-2206

70. Penn-Nicholson A et al (2015) Safety and immunogenicity of candidate vaccine M72/AS01E in adolescents in a TB endemic setting. Vaccine 33(32):4025-4034

71. Thacher EG et al (2014) Safety and immunogenicity of the M72/ AS01 candidate tuberculosis vaccine in HIV-infected adults on combination antiretroviral therapy: a phase I/II, randomized trial. AIDS 28(12):1769-1781

72. Kumarasamy $\mathrm{N}$ et al (2018) Long-term safety and immunogenicity of the M72/AS01E candidate tuberculosis vaccine in HIVpositive and -negative Indian adults: results from a phase II randomized controlled trial. Medicine (Baltimore) 97(45):S

73. Van Der Meeren O et al (2018) Phase 2b controlled trial of M72/ AS01E vaccine to prevent tuberculosis. N Engl J Med 379(17): $1621-1634$

74. Cardona PJ (2006) RUTI: a new chance to shorten the treatment of latent tuberculosis infection. Tuberculosis (Edinb) 86(3-4):273289

75. Vilaplana C et al (2010) Double-blind, randomized, placebocontrolled phase I clinical trial of the therapeutical antituberculous vaccine RUTI. Vaccine 28(4):1106-1116

76. Nell AS et al (2014) Safety, tolerability, and immunogenicity of the novel antituberculous vaccine RUTI: randomized, placebocontrolled phase II clinical trial in patients with latent tuberculosis infection. PLoS One 9(2):e89612

77. von Reyn CF et al (2010) Prevention of tuberculosis in Bacille Calmette-Guerin-primed, HIV-infected adults boosted with an inactivated whole-cell mycobacterial vaccine. AIDS 24(5):675685

78. Lahey $\mathrm{T}$ et al (2016) Immunogenicity and protective efficacy of the DAR-901 booster vaccine in a murine model of tuberculosis. PLoS One 11(12):e0168521

79. von Reyn CF et al (2017) Safety and immunogenicity of an inactivated whole cell tuberculosis vaccine booster in adults primed with BCG: a randomized, controlled trial of DAR-901. PLoS One 12(5):e0175215

80. Masonou $\mathrm{T}$ et al (2019) CD4+ T cell cytokine responses to the DAR-901 booster vaccine in BCG-primed adults: a randomized, placebo-controlled trial. PLoS One 14(5):e0217091

81. Craig SR et al (2018) Altruism, scepticism, and collective decision-making in foreign-born U.S. residents in a tuberculosis vaccine trial. BMC Public Health 18(1):535

82. Sharma $P$ et al (1999) Disabilities in multibacillary leprosy following multidrug therapy with and without immunotherapy with Mycobacterium w antileprosy vaccine. Int J Lepr Other Mycobact Dis 67(3):250-258

83. Sharma $P$ et al (2005) Immunoprophylactic effects of the antileprosy $\mathrm{Mw}$ vaccine in household contacts of leprosy patients: clinical field trials with a follow up of 8-10 years. Lepr Rev 76(2):127-143
84. Sharma $\mathrm{P}$ et al (2000) Mycobacterium w vaccine, a useful adjuvant to multidrug therapy in multibacillary leprosy: a report on hospital based immunotherapeutic clinical trials with a follow-up of 1-7 years after treatment. Lepr Rev 71(2):179-192

85. Guleria I, Mukherjee R, Kaufmann SH (1993) In vivo depletion of CD4 and CD8 T lymphocytes impairs Mycobacterium w vaccineinduced protection against $\mathrm{M}$. tuberculosis in mice. Med Microbiol Immunol 182(3):129-135

86. Gupta A et al (2012) Protective efficacy of Mycobacterium indicus pranii against tuberculosis and underlying local lung immune responses in guinea pig model. Vaccine 30(43):6198-6209

87. Patel N, Deshpande MM, Shah M (2002) Effect of an immunomodulator containing Mycobacterium w on sputum conversion in pulmonary tuberculosis. J Indian Med Assoc 100(3):191-193

88. Patel N, Trapathi SB (2003) Improved cure rates in pulmonary tuberculosis category II (retreatment) with mycobacterium w. J Indian Med Assoc 101(11):680 682

89. Groschel MI et al (2014) Therapeutic vaccines for tuberculosis-a systematic review. Vaccine 32(26):3162-3168

90. Gonzalo-Asensio J et al (2017) MTBVAC: attenuating the human pathogen of tuberculosis (TB) toward a promising vaccine against the TB epidemic. Front Immunol 8:1803

91. Aguilo $\mathrm{N}$ et al (2016) MTBVAC vaccine is safe, immunogenic and confers protective efficacy against Mycobacterium tuberculosis in newborn mice. Tuberculosis (Edinb) 96:71-74

92. Marinova D et al (2017) MTBVAC from discovery to clinical trials in tuberculosis-endemic countries. Expert Rev Vaccines 16(6):565-576

93. Spertini F et al (2015) Safety of human immunisation with a liveattenuated Mycobacterium tuberculosis vaccine: a randomised, double-blind, controlled phase I trial. Lancet Respir Med 3(12): 953-962

94. World Health Organization (1995) Global tuberculosis program and global program on vaccine: statement on $\mathrm{BCG}$ revaccination for the prevention of tuberculosis. WHO, Geneva

95. World Health Organization (2004) BCG vaccine. WHO position paper. WHO, Geneva

96. World Health Organization (2018) BCG vaccines: WHO position paper-February 2018. WHO, Geneva

97. World Health Organization (2007) Revised BCG vaccination guidelines for infants at risk for HIV infection. WHO, Geneva

98. Husain AA et al (2011) Effect of repeat dose of BCG vaccination on humoral response in mice model. Indian J Exp Biol 49(1):7-10

99. Husain AA et al (2015) Comparative evaluation of booster efficacies of BCG, Ag85B, and Ag85B peptides based vaccines to boost BCG induced immunity in BALB/c mice: a pilot study. Clin Exp Vaccine Res 4(1):83-87

100. Parlane NA et al (2014) Revaccination of cattle with bacille Calmette-Guerin two years after first vaccination when immunity has waned, boosted protection against challenge with Mycobacterium bovis. PLoS One 9(9):e106519

101. Kashyap RS et al (2010) Assessment of immune response to repeat stimulation with BCG vaccine using in vitro PBMC model. J Immune Based Ther Vaccines 8:3

102. Nemes E et al (2018) Prevention of M. tuberculosis infection with H4:IC31 vaccine or BCG revaccination. N Engl J Med 379(2): 138-149

103. Nieuwenhuizen NE et al (2017) The recombinant Bacille Calmette-Guerin vaccine VPM1002: ready for clinical efficacy testing. Front Immunol 8:1147

104. Hamon MA et al (2012) Listeriolysin O: the Swiss army knife of Listeria. Trends Microbiol 20(8):360-368

105. Shaughnessy LM et al (2006) Membrane perforations inhibit lysosome fusion by altering $\mathrm{pH}$ and calcium in Listeria monocytogenes vacuoles. Cell Microbiol 8(5):781-792 
106. Reyrat JM, Berthet FX, Gicquel B (1995) The urease locus of Mycobacterium tuberculosis and its utilization for the demonstration of allelic exchange in Mycobacterium bovis bacillus Calmette-Guerin. Proc Natl Acad Sci U S A 92(19):8768-8772

107. Gordon AH, Hart PD, Young MR (1980) Ammonia inhibits phagosome-lysosome fusion in macrophages. Nature 286(5768): 79-80

108. Kaufmann SH et al (2014) The BCG replacement vaccine VPM1002: from drawing board to clinical trial. Expert Rev Vaccines 13(5):619-630

109. Grode L et al (2013) Safety and immunogenicity of the recombinant BCG vaccine VPM1002 in a phase 1 open-label randomized clinical trial. Vaccine 31(9): 1340-1348

110. Loxton AG,Knaul JK,Grode L,Gutschmidt A,Meller C,Eisele B, Johnstone H,van der Spuy G,Maertzdorf J,Kaufmann SHE, Hesseling AC,Walzl G,Cotton MF., Safety and Immunogenicity of the Recombinant Mycobacterium bovis BCG Vaccine VPM1002 in HIV-Unexposed Newborn Infants in South Africa. Clin Vaccine Immunol. 2017 Feb 6;24(2). pii: e00439-16. https:// doi.org/10.1128/CVI.00439-16. Print 2017 Feb

111. Lindenstrom $\mathrm{T}$ et al (2013) Control of chronic mycobacterium tuberculosis infection by CD4 KLRG1- IL-2-secreting central memory cells. J Immunol 190(12):6311-6319

112. Grode L et al (2005) Increased vaccine efficacy against tuberculosis of recombinant Mycobacterium bovis bacille CalmetteGuerin mutants that secrete listeriolysin. J Clin Invest 115(9): 2472-2479

113. Desel C et al (2011) Recombinant BCG DeltaureC hly+ induces superior protection over parental BCG by stimulating a balanced combination of type 1 and type 17 cytokine responses. J Infect Dis 204(10):1573-1584

114. Vogelzang A et al (2014) Central memory CD4+ T cells are responsible for the recombinant Bacillus Calmette-Guerin DeltaureC::hly vaccine's superior protection against tuberculosis. J Infect Dis 210(12):1928-1937

115. Gengenbacher $\mathrm{M}$ et al (2016) Post-exposure vaccination with the vaccine candidate Bacillus Calmette-Guerin DeltaureC::hly induces superior protection in a mouse model of subclinical tuberculosis. Microbes Infect 18(5):364-368

116. Gengenbacher M, Nieuwenhuizen N, Vogelzang A, Liu H, Kaiser P, Schuerer S, Lazar D, Wagner I, Mollenkopf HJ, Kaufmann SH., Deletion of nuoG from the Vaccine Candidate Mycobacterium bovis BCG DeltaureC::hly Improves Protection against Tuberculosis. mBio.2016 May 24;7(3). pii: e00679-16. https:// doi.org/10.1128/mBio.00679-16

117. Velmurugan K et al (2013) Nonclinical development of BCG replacement vaccine candidates. Vaccines (Basel) 1(2):120-138

118. Irwin SM et al (2005) Tracking antigen-specific CD8 T lymphocytes in the lungs of mice vaccinated with the Mtb $72 \mathrm{~F}$ polyprotein. Infect Immun 73(9):5809-5816

119. Brandt L et al (2004) The protective effect of the Mycobacterium bovis BCG vaccine is increased by coadministration with the Mycobacterium tuberculosis 72-kilodalton fusion polyprotein $\mathrm{Mtb} 72 \mathrm{~F}$ in $\mathrm{M}$. tuberculosis-infected guinea pigs. Infect Immun 72(11):6622-6632

120. Tsenova L et al (2006) Evaluation of the Mtb72F polyprotein vaccine in a rabbit model of tuberculous meningitis. Infect Immun 74(4):2392-2401

121. Reed SG et al (2009) Defined tuberculosis vaccine, Mtb72F/ AS02A, evidence of protection in cynomolgus monkeys. Proc Natl Acad Sci U S A 106(7):2301-2306

122. Cooper $\mathrm{AM}$ et al (2009) $\mathrm{T}$ cells in mycobacterial infection and disease. Curr Opin Immunol 21(4):378-384

123. Calderon VE et al (2013) A humanized mouse model of tuberculosis. PLoS One 8(5):e63331
124. Commandeur S et al (2014) The in vivo expressed Mycobacterium tuberculosis (IVE-TB) antigen Rv2034 induces $\mathrm{CD} 4{ }^{+}$T-cells that protect against pulmonary infection in HLADR transgenic mice and Guinea pigs. Vaccine 32(29):3580-3588

125. Nusbaum RJ et al (2016) Pulmonary tuberculosis in humanized mice infected with HIV-1. Sci Rep 6:21522

126. Lanoix JP et al (2015) Sterilizing activity of pyrazinamide in combination with first-line drugs in a $\mathrm{C} 3 \mathrm{HeB} / \mathrm{FeJ}$ mouse model of tuberculosis. Antimicrob Agents Chemother 60(2):1091-1096

127. Lanoix JP et al (2015) Heterogeneous disease progression and treatment response in a $\mathrm{C} 3 \mathrm{HeB} / \mathrm{FeJ}$ mouse model of tuberculosis. Dis Model Mech 8(6):603-610

128. Aagaard C et al (2011) A multistage tuberculosis vaccine that confers efficient protection before and after exposure. Nat Med 17(2):189-194

129. Jacobs RE et al (2015) Reactivation of pulmonary tuberculosis during cancer treatment. Int J Mycobacteriol 4(4):337-340

130. Kashino SS et al (2008) Guinea pig model of Mycobacterium tuberculosis latent/dormant infection. Microbes Infect 10(14 15):1469-1476

131. Ordway DJ et al (2010) Evaluation of standard chemotherapy in the guinea pig model of tuberculosis. Antimicrob Agents Chemother 54(5):1820-1833

132. Clark S et al (2014) Animal models of tuberculosis: guinea pigs. Cold Spring Harb Perspect Med 5(5):a018572

133. Converse PJ et al (1996) Cavitary tuberculosis produced in rabbits by aerosolized virulent tubercle bacilli. Infect Immun 64(11): 4776-4787

134. Zhang $\mathrm{G}$ et al (2010) Evaluation of mycobacterial virulence using rabbit skin liquefaction model. Virulence 1(3):156-163

135. Dannenberg AM Jr (2009) Liquefaction and cavity formation in pulmonary TB: a simple method in rabbit skin to test inhibitors. Tuberculosis (Edinb) 89(4):243-247

136. Sun $\mathrm{H}$ et al (2012) Effects of immunomodulators on liquefaction and ulceration in the rabbit skin model of tuberculosis. Tuberculosis (Edinb) 92(4):345-350

137. Peng X et al (2015) Rabbit models for studying human infectious diseases. Comp Med 65(6):499-507

138. Manabe YC et al (2008) The aerosol rabbit model of TB latency, reactivation and immune reconstitution inflammatory syndrome. Tuberculosis (Edinb) 88(3):187-196

139. Rahyussalim AJ et al (2016) New bone formation in tuberculousinfected vertebral body defect after administration of bone marrow stromal cells in rabbit model. Asian Spine J 10(1):1-5

140. Scanga CA et al (1999) Reactivation of latent tuberculosis: variations on the Cornell murine model. Infect Immun 67(9):45314538

141. Izzo AA et al (2015) A novel MVA-based multiphasic vaccine for prevention or treatment of tuberculosis induces broad and multifunctional cell-mediated immunity in mice and primates. PLoS One 10(11):e0143552

142. Pena JC et al (2015) Monkey models of tuberculosis: lessons learned. Infect Immun 83(3):852-862

143. Phuah $\mathrm{J}$ et al (2016) Effects of B cell depletion on early Mycobacterium tuberculosis infection in cynomolgus macaques. Infect Immun 84(5): 1301-1311

144. Lin PL et al (2009) Quantitative comparison of active and latent tuberculosis in the cynomolgus macaque model. Infect Immun 77(10):4631-4642

145. Diedrich CR et al (2010) Reactivation of latent tuberculosis in cynomolgus macaques infected with SIV is associated with early peripheral T cell depletion and not virus load. PLoS One 5(3): e9611

146. Kupferschmidt K (2011) Infectious disease. Taking a new shot at a TB vaccine. Science 334(6062):1488-1490 
147. McShane $\mathrm{H}$ et al (2004) Recombinant modified vaccinia virus Ankara expressing antigen 85A boosts BCG-primed and naturally acquired antimycobacterial immunity in humans. Nat Med 10(11): 1240-1244

148. Verreck FA et al (2009) MVA.85A boosting of BCG and an attenuated, phoP deficient M. tuberculosis vaccine both show protective efficacy against tuberculosis in rhesus macaques. PLoS One 4(4):e5264

149. Beveridge NE et al (2007) Immunisation with BCG and recombinant MVA85A induces long-lasting, polyfunctional Mycobacterium tuberculosis-specific CD4+ memory T lymphocyte populations. Eur J Immunol 37(11):3089-3100

150. Minassian AM et al (2011) A phase I study evaluating the safety and immunogenicity of MVA85A, a candidate TB vaccine, in HIV-infected adults. BMJ Open 1(2):e000223

151. Odutola AA et al (2012) A new TB vaccine, MVA85A, induces durable antigen-specific responses 14 months after vaccination in African infants. Vaccine 30(38):5591-5594

152. Pathan AA et al (2012) Effect of vaccine dose on the safety and immunogenicity of a candidate TB vaccine, MVA85A, in BCG vaccinated UK adults. Vaccine 30(38):5616-5624

153. Sander CR et al (2009) Safety and immunogenicity of a new tuberculosis vaccine, MVA85A, in Mycobacterium tuberculosisinfected individuals. Am J Respir Crit Care Med 179(8):724-733

154. Scriba TJ et al (2010) Modified vaccinia Ankara-expressing $\mathrm{Ag} 85 \mathrm{~A}$, a novel tuberculosis vaccine, is safe in adolescents and children, and induces polyfunctional CD4+ T cells. Eur J Immunol 40(1):279-290

155. Scriba TJ et al (2011) Dose-finding study of the novel tuberculosis vaccine, MVA85A, in healthy BCG-vaccinated infants. J Infect Dis 203(12):1832-1843
156. Scriba TJ et al (2012) A phase IIa trial of the new tuberculosis vaccine, MVA85A, in HIV- and/or Mycobacterium tuberculosisinfected adults. Am J Respir Crit Care Med 185(7):769-778

157. Tameris MD et al (2013) Safety and efficacy of MVA85A, a new tuberculosis vaccine, in infants previously vaccinated with BCG: a randomised, placebo-controlled phase $2 \mathrm{~b}$ trial. Lancet 381(9871): 1021-1028

158. Cole ST et al (1998) Deciphering the biology of Mycobacterium tuberculosis from the complete genome sequence. Nature 393(6685):537-544

159. Kaufmann SH (2011) Fact and fiction in tuberculosis vaccine research: 10 years later. Lancet Infect Dis 11(8):633-640

160. Bhatt K et al (2015) Quest for correlates of protection against tuberculosis. Clin Vaccine Immunol 22(3):258-266

161. Barker LF et al (2009) Tuberculosis vaccine research: the impact of immunology. Curr Opin Immunol 21(3):331-338

162. Cooper AM (2009) T cells in mycobacterial infection and disease. Curr Opin Immunol 21(4):378-384

163. Williams A, Hall Y, Orme IM (2009) Evaluation of new vaccines for tuberculosis in the guinea pig model. Tuberculosis (Edinb) 89(6):389-397

164. Reed SG et al (2003) Prospects for a better vaccine against tuberculosis. Tuberculosis (Edinb) 83(1-3):213-219

165. Fennelly KP et al (2012) Variability of infectious aerosols produced during coughing by patients with pulmonary tuberculosis. Am J Respir Crit Care Med 186(5):450-457

Publisher's note Springer Nature remains neutral with regard to jurisdictional claims in published maps and institutional affiliations. 